Series A

I. MATHEMATICA

504

\title{
ON DISCRETE GROUPS OF THE UNIT DISK AND THEIR ISOMORPHISMS
}

BY

PEKKA TUKIA 
Copyright (C) 1972 by

Academia Scientiarum Fennica

ISBN $951-41-0030-1$

Communicated 13 September 1971 by K. I. Virtanen 


\section{PREFACE}

I wish to express my gratitude to my teacher, Professor K. I. Virtanen, who suggested the subject from which this work emerged. I wish also to thank Dr. Kalevi Suominen for the trouble he has taken in improving the manuscript.

This work has been financially supported by a research grant of the University of Helsinki and by another from the Finnish Academy of Sciences.

Helsinki, September 1971

Pekka Tukia 


\section{Introduction}

The subject matter of this paper consists of the study of certain groups of conformal transformations of the unit disk $|z|<1$ of the complex plane and specially isomorphisms between them. These groups are usually called discrete groups of conformal mappings of the unit disk, even if we have adopted the general standpoint (cf. definition 2.1) that a discrete group may contain indirectly conformal mappings and, in particular, reflections, which is not always the case. If a group of this kind does not contain indirectly conformal mappings, then it is usually called a Fuchsian group.

The starting point of the present investigation is a well-known result of Nielsen in [5]. Nielsen considered groups $F$ and $F^{\prime}$ such that the unit disk $E$ is the universal covering space of the factor spaces $E / F$ and $E / F^{\prime}$, which were in addition assumed to be compact surfaces. He showed that if $\varphi: F \rightarrow F^{\prime}$ is any isomorphism then it is induced by a homeomorphism $f: E \rightarrow E$ such that $f(T(x))=\varphi(T)(f(x))$ for $x \in E$ and $T \in F$. Later this result has been proved for any discrete groups $F$ and $F^{\prime}$ of the unit disk such that $E / F$ and $E / F^{\prime}$ are compact. (Cf. Zieschang [6] and Macbeath [3]). On the other hand, if the factor spaces are not compact, it is easy to see that there are isomorphisms that are not induced by a homeomorphism of the unit disk.

The aim of this investigation was to obtain a precise condition for an isomorphism $\varphi: F \rightarrow F^{\prime}$ of two discrete groups to be induced by a homeomorphism of the unit disk in case the factor spaces $E / F$ and $E / F^{\prime}$ are not compact. This has been done, with some limitations on the groups $F$ and $F^{\prime}$, in theorem 3.6. According to this theorem $\varphi$ is induced by a homeomorphism of the unit disk if and only if it preserves a cortain relation, called the relation of baing crossed. Two hrperbolic el ments $T_{1}$ and $T_{2}$ are said to be crossed if their axes, i.e. the circle ares in the wnit disk orthogonal to the unit circle connecting the fixed points of $T_{1}$ and $T_{2}$, intersect. $\varphi$ preserves the relation of being crossed if, when $T_{1}$ and $T_{2}$ are two hyperbolic elements of $F, T_{1}$ and $T_{2}$ are crossed if and only if $\varphi\left(T_{1}\right)$ and $\varphi\left(T_{2}\right)$ are crossed.

There are certain by-products. First of all, if $F$ is a discrete group of the unit disk not containing reflections such that $E / F$ is non-compact, then $F$ is a free product of cyclic groups. In case $F$ contains reflections 
it is shown that $F$ is a skew product of $\mathbf{Z}_{2}$ and a free product of cyclic groups. (Cf. corollary 2.8.1). In corollary 3.6.1 we show that if $F$ and $F^{\prime}$ are discrete groups containing reflections such that $E / F$ and $E / F^{\prime}$ are compact, then any isomorphism $F \rightarrow F^{\prime}$ is induced by a homeomorphism. This result has been known previously (cf. Macbeath [3]) but the proof has involved some results of the theory of Teichmüller spaces that we dispense with. Finally, we show that if $F^{\prime}$ and $F^{\prime}$ are groups of the first kind, i.e. their limit points are dense in the unit circle, then any homeomorphism of the unit disk inducing an isomorphism $F \rightarrow F^{\prime}$ may be extended to a homeomorphism of the closed unit disk $|z| \leq 1$ of the complex plane. (Cf. corollary 3.5.1).

\section{Preliminary notions and the isometric circle}

A. Preliminary notions about the conformal mappings of the unit circle. We are concerned in this work with discrete groups of conformal mappings of the unit circle $E=\{z \in \mathbf{C}:|z|<1\}$ of the complex plane $\mathbf{C}$. Hence, to begin with, we shall establish a few basic notations and facts about the conformal mappings of the unit disk. We shall use the word 》conformal» in a sense that includes both directly and indirectly conformal mappings. Thus a conformal mapping may not preserve orientation.

We shall use the following notations:

$$
\begin{array}{ll}
E=\{z \in \mathbf{C}:|z|<1\} & \text { the open unit disk } \\
E^{1}=\{z \in \mathbf{C}:|z| \leq 1\} & \text { the closed unit disk } \\
S^{1}=\{z \in \mathbf{C}:|z|=1\} & \text { the unit circle }
\end{array}
$$

If $T$ is a conformal mapping of the open unit disk, it can be uniquely extended to a conformal mapping of the Riemann sphere. We shall not distinguish between $T$ and its extension to the Riemann sphere. We agree to call a conformal mapping $T$ of the Riemann sphere a conformal mapping of the unit disk if it leaves $E$ invariant.

If $T$ is a directly conformal mapping of the unit disk, different from the identity, there are three possibilities present:

(a) $T$ is elliptic and leaves one point fixed in $E$. We denote this point by $\operatorname{Fix}(T)$. In the cases of interest, the order of $T$ is finite, since otherwise no group of conformal mappings containing $T$ can be discrete.

(b) $T$ is hyperbolic and has two fixed points on $S^{1}$, denoted $P(T)$ and $N(T)$ such that the following equations are true:

$$
\begin{aligned}
& \lim _{n \rightarrow \infty} T^{n}(z)=P(T), z \in E \\
& \lim _{n \rightarrow-\infty} T^{n}(z)=N(T), z \in E .
\end{aligned}
$$


The order of $T$ is infinite. The closed arc of the circle orthogonal to $S^{1}$ contained in $E^{1}$ and having $P(T)$ and $N(T)$ as the endpoints is denoted by

$$
\operatorname{Ax}(T) \text {. }
$$

(c) $T$ is parabolic. In this case $T$ has one fixed point on $S^{1}$. We denote this point by $P(T)$ or by $N(T)$. The equations (1) are still valid. The order of $T$ is infinite and we denote

$$
\operatorname{Ax}(T)=\{P(T)\}=\{N(T)\} .
$$

If $T$ is an indirectly conformal mapping of the unit disk, there are basically two alternatives:

(d) $T$ is a reflection in a circle $O$ orthogonal to $S^{1}$. We set

$$
\operatorname{Ax}(T)=O \cap E^{1}
$$

$T$ satisfies the relation $T^{2}=i d$.

(e) $T=T^{\prime} S=S T^{\prime}$ where $T^{\prime}$ is a hyperbolic, directly conformal mapping of the unit disk and $S$ is a reflection on $\operatorname{Ax}\left(T^{\prime}\right)$. In this case $T$ has two fixed points in $S^{1}$, denoted $P(T)$ and $N(T)$, in such a way that equations (1) hold true. The arc of the circle orthogonal to $S^{1}$, denoted $\mathrm{Ax}(T)$, is defined as in (2). The order of $T$ is infinite.

The word hyperbolic is also used in the case (e). Hence, an indirectly conformal mapping of the unit disk that has no fixed points in $E$ is necessarily hyperbolic, of the type $(e)$.

B. The isometric circle. The concept of the isometric circle of a conformal mapping turns out to be quite central in our later developements. Hence we establish here the properties of the isometric circle needed.

The isometric circle of a conformal mapping $T$ is defined by the differential equation (3):

$$
|d T|=|d z|
$$

An isometric circle of a conformal mapping may not exist nor be uniquely determined. However, if $T$ is a conformal mapping of the unit disk, an isometric circle always exists, but it may not be unique. It is miquely determined by (3) except for the following three cases:

(i) $T$ is the identity.

(ii) $T$ is elliptic and $\operatorname{Fix}(T)$ is the origin.

(iii) $T$ is a reflection such that $\mathrm{Ax}(T)$ passes through the origin.

In all these cases the equation (3) is true on any circle and thus, any circle is an isometric circle for $T$.

We shall need the following facts about the isometric circle of a conformal mapping: 
1.1. Proposition. Let $O_{1}$ and $O_{2}$ be two circles orthogonal to the unit circle and having an equal diameter. Then there is a unique directly conformal mapping of the unit disk $T$ and an indirectly conformal mapping of the unit disk $S$ such that the isometric circle of $S$ and $T$ is $O_{1}$ and that of $S^{-1}$ and $T^{-1}$ is $O_{2}$, and such that $T\left(O_{1}\right)=O_{2}$ and $S\left(O_{1}\right)=O_{2}$. Besides we have:

(a) If $O_{1}=O_{2}, T$ is elliptic of order two and $\operatorname{Fix}(T)$ is the middle point of the arc $O_{1} \cap E^{1}$.

(b) If $\{x\}=O_{1} \cap O_{2} \cap E$, then $T$ is elliptic and $\operatorname{Fix}(T)=x$.

(c) If $O_{1} \cap O_{2} \cap E=\varnothing$, then $T$ is hyperbolic or parabolic and $s$ is hyperbolic. The fixed points of $S$ and $T$ are inside or on the circles $O_{1}$ and $\mathrm{O}_{2}$.

The conformal mappings $S$ and $T$ of proposition 1.1 are constructed by combining suitable reflections of the Riemann sphere. We leave the explicit construction to the reader.

The following proposition shows that it is always possible to construct elliptic transformations of the unit disk of a given order whose isometric circles satisfy some conditions given beforehand:

1.2 Proposition. Let $I$ be a closed interval of $S^{1}$ and $n \geq 2$ a given integer. Then there are two circles orthogonal to $S^{1}$, denoted $O_{1}$ and $O_{2}$, and a conformal mapping $T$ of the unit disk such that

$$
I=\left(\bar{O}_{1} \cup \bar{O}_{2}\right) \cap S^{1}
$$

when the closure of the interior of a circle $K$ is denoted by $\bar{K}$. The mapping $T$ is such that $T$ is a rotation through the angle $2 \pi / n$ and the isometric circle of $T$ is $O_{1}$ and that of $T^{-1}$ is $O_{2}$, and $T\left(O_{1}\right)=O_{2}$.

This proposition is also obvious. It may be remarked that the circles $O_{1}$ and $O_{2}$ are uniquely determined.

The fundamental property of the isometric circle of a conformal mapping $T$ is that $T$ is expansive inside it and contractive outside it. We use this property in the form stated by the proposition 1.3. The proof is again obvious and is left to the reader.

1.3 Proposition. Let $T$ be a conformal mapping of the unit disk with a unique isometric circle. Then if $d>0$ is given, there is a real number $k, o<k<1$, such that

$$
l(T(I))<k l(I)
$$

when $I$ is an interval of $S^{1}$ outside the isometric circle of $T$ and the length of an interval $J$ of $S^{1}$ is denoted by $l(J)$ and $l(I) \geq d$. If $T$ is a rotation through the angle $2 \pi / n$, then the constant $k$ may be chosen so that 


$$
l\left(T^{i}(I)\right)<k l(I)
$$

where $1 \leq i<n$ and $I$ is also outside the isometric circle of $T^{-1}$.

Propositions 1.1-1.3 summarize the facts we need about isometric circles of conformal mappings. The reader wishing a more comprehensive treatment is referred to Ford [2], where also proofs of some of the above propositions can be found.

C. Miscellaneous. To conclude this chapter we prove a proposition that allows us to infer, when $F$ is a discrete group of conformal mappings of the unit disk such that $\operatorname{Fix}(F)=\{P(T): T \in F$, the order of $T$ is infinite $\}$ is dense in $S^{1}, I$ and $J$ arbitrary intervals of $S^{1}$, the existence of an element $T$ of $F$ such that $P(T) \in I$ and $N(T) \in J$.

1.4. Proposition. Let $S$ and $T$ be two conformal mappings of the unit disk, both having infinite order and no common fixed points. Then if $U$ is a neighbourhood of $P(T)$ in $S^{1}$ and $V$ a neighbourhood of $P(S)$ in $S^{1}$, then for some integer $n>o P\left(T^{n} S^{-n}\right) \in U$ and $N\left(T^{n} S^{-n}\right) \in V$.

Proof: Choose $n$ so large that there are circles $A, A^{\prime}, B, B^{\prime}$ external to each others (or tangent externally to each others) such that $P(T) \in \bar{A}$, $N(T) \in \bar{A}^{\prime}, \quad P(S) \in \bar{B}, \quad N(S) \in \bar{B}^{\prime}$ (denoting the closure of the interior of a circle $K$ by $\bar{K})$, and such that $T^{n}\left(A^{\prime}\right)=A$ and $S^{n}\left(B^{\prime}\right)=B$. Besides we may suppose that $\bar{A} \cap S^{1} \subset U$ and $\bar{B} \cap S^{1} \subset V$. Then $T^{n} S^{-n}(\bar{A}) \subset \bar{A}$ and $S^{n} T^{-n}(\bar{B}) \subset \bar{B}$. This proves the proposition.

\section{Discrete groups of conformal mappings and pointed surfaces}

A. Discrete groups of simply connected Riemann surfaces. In this section we consider a discrete group of a simply connected Riemann surface $X$ and the resulting canonical projection $p: X \rightarrow X / F=S$. With the help of the projection $p$ we define a mapping $n: S \rightarrow \mathbf{N}$. This leads us to the concept of a pointed surface in the beginning of the next section.

As is well known, every simply connected Riemann surface is conformally equivalent to one of the following Riemann surfaces:

$$
\begin{aligned}
& S^{2} \text { the Riemann sphere } \\
& \text { C the complex plane } \\
& E \text { the open unit disk }
\end{aligned}
$$

Let $X$ denote one of these surfaces and let $F$ be a group of conformal equivalences of $X$ onto itself.

2.1. Definition. The group $F$ is a discrete group of $X$ if, given $z \in X$, there is a finite subgroup $F_{z}$ of $F$ and a neighbourhood $U$ of $z$ such that 


$$
\begin{aligned}
U \cap T(U) & =\varnothing \text { for } T \in F \backslash F_{z} \\
T(z) & =z \text { for } T \in F_{z} .
\end{aligned}
$$

The group $F_{z}$ that is obviously uniquely determined by these conditions is called the stabilizer of $z$.

It is not difficult to see that the stabilizer of a point $z \in X$ must be one of the following groups:

(a) $F_{z}=\left\{i d_{X}\right\}$. The stabilizer $F_{z}$ is trivial.

(b) $F_{z}$ is the group generated by a rotation through the angle $2 \pi / n$. The stabilizer $F_{z}$ is the rotation group of degree n.

(c) $F_{z}$ is the group generated by a reflection $T$ satisfying the relation $T^{2}=i d$. The stabilizer $F_{z}$ is the reflection group.

(d) $F_{z}$ is a group of order $2 n$ having as generators two reflections $S$ and $T$ such that $S T$ is a rotation through the angle $2 \pi / n$. The stabilizer $F_{z}$ is the dihedral group of degree $n$.

Note that in $(d) F_{z}$ is as an abstract group the group generated by $S$ and $T$ and having the relations

$$
S^{2}=T^{2}=(S T)^{n}=1 .
$$

The factor space $S=X / F$ is a bordered surface that is naturally endowed with a conformal structure. Its boundary is empty if and only if there are no reflections in $F$. Let $p: X \rightarrow S$ be the canonical projection. It is a local conformal equivalence at a point $z \in X$ if and only if the stabilizer $F_{z}$ of $z$ is trivial. At other points of $X$ the local behaviour of the mapping $p$ may be described as follows:

(i) If $F_{z}$ is the rotation group of degree $n$, then $p$ is in in suitable local coordinates of the form

$$
z \rightarrow z^{n}
$$

In this case we say that $p$ is of $d$ e gree $n$ at $z$. This includes also the case where $F_{z}$ is trivial, if we set $n=1$ in this case.

To study the local behaviour of $p$ at points where $p(z)$ lies on the boundary of $S$, i.e. $F_{z}$ is a reflection group or a dihedral group, we define a special mappirg $\tau: \mathbf{C} \rightarrow \mathbf{C}$ such that

$$
\tau(x+i y)=x+i y^{\prime}, x, y \in \mathbf{R} .
$$

(ii) If $F_{z}$ is the reflection group or dihedral group of degree $n$, then, in suitable local coordinates, $p$ is of the form

$$
z \rightarrow \tau\left(z^{n}\right)
$$

where $n=1$ if $F_{z}$ is the reflection group and $n>1$ if $F_{z}$ is the dihedral group of degrce $n$. In this case we say $p$ is of dihedral degree $n$. 
Above we have associated with each discrete group of conformal mappings of a simply connected Riemann surface $X$ a bordered Riemann surface $S$ and a projection $p: X \rightarrow S$. The local behaviour of $p$ will be defined by a mapping $n: S \rightarrow \mathbf{N}$, where $\mathbf{N}$ is the set of the natural numbers (zero exempted), such that

$$
n(x)=k
$$

if the degree or dihedral degree of $p$ at a point $z \in p^{-1}(x)$ is $k$. This is easily seen to be independent of the choice of $z$. The set where $n>1$ is a discrete subset of $S$.

Finally we make an observation to which we have an occasion to refer in connexion of the proposition 2.4. The property ( $i i i$ ) of the projection $p: X \rightarrow X / F=S$ suggests that the situation we are considering is a generalization of the case where $p$ is the covering projection of the universal covering surface of a surface $S$. This analogy, too, is clarified in the proposition 2.4 .

(iii) If $x \in S$ there is a neighbourhood $U$ of $x$ such that the components of $p^{-1}(U)$ are $U_{i}, i \in I$, and restriction of $p$ to $U_{i}, i \in I$, is of the form

$$
\begin{aligned}
& z \rightarrow z^{n(x)} \quad \text { or } \\
& z \rightarrow \tau\left(z^{n(x)}\right)
\end{aligned}
$$

according to whether $x$ lies on the boundary of $S$ or is an interior point of $S$.

So we shall call the canonical projection $p: X \rightarrow S$ a covering projection, if this seems appropriate.

B. Pointed surfaces. The above considerations give rise to the following definition:

2.2 Definition. A pointed surface is a pair $(S, n)$ where $S$ is a bordered surface with countable basis and $n: S \rightarrow \mathbf{N}$ mapping such that the set $|n|=$ $\{x \in S: n(x)>1\}$, called the support of $n$, is discrete. In case $S$ has a conformal structure the pair $(S, n)$ is called a pointed Riemann surface.

Particularly if $(S, n)$ is the pointed Riemann surface defined above by the discrete group $F$ of a simply connected Riemann surface $X$, we denote

$$
(S, n)=\left(S_{F}, n_{F}\right)=\left(S_{p}, n_{p}\right)
$$

where $p$ is the covering projection $X \rightarrow S$.

We next state the inverse problem:

2.3 Problem. Given a pointed Riemann surface $(S, n)$, is it possible to represent it in the form (*) for suitable $X$ and $F$ ?

It is quite natural to define two pointed surfaces $(S, n)$ and $\left(S^{\prime}, n^{\prime}\right)$ homeomorphic if there exists a homeomorphism $f: S \rightarrow S^{\prime}$ such that 
$n^{\prime}(f(x))=n(x)$. It is quite clear that the solution of the problem 2.3 depends only on the homeomorphism class of the pointed Riemann surface $(S, n)$ with this definition of homeomorphisms of pointed surfaces. To this end we prove proposition 2.4, but before we do this we define, corresponding to each pointed Riemann surface $(S, n)$, a class of mappings $p: X \rightarrow S$, where $X$ is some Riemann surface (without boundary), that generalizes the situation where $p$ is the covering projection of the covering surface $X$. Since proposition 2.4 is the only place where this class of mappings is used, this may seem unnecessary, but proposition 2.4 is most naturally expressed in terms of these mappings, to be called covering projections $p: X \rightarrow(S, n)$.

Let $X$ be a connected Riemann surface (without boundary), $(S, n)$ a pointed Riemann surface. Then a mapping $p: X \rightarrow S$ is a covering projection $p: X \rightarrow(S, n)$ if it satisfies the following condition:

Given a point $x \in S$, there is a neighbourhood $U$ of $x$ such that the components of $p^{-1}(U)$ are $U_{i} . i \in I$, and that the restriction of $p$ to $U_{i}$ is of the form

$$
\begin{aligned}
& z \rightarrow z^{k_{i}} \quad \text { if } x \text { is an interior point of } S \\
& z \rightarrow \tau\left(z^{k_{i}}\right) \quad \text { if } x \text { is a boundary point of } S
\end{aligned}
$$

for $i \in I$, and where $k_{i}$ divides $n(x)$.

2.4. Proposition. Let $X_{i}$ be a Riemann surface, $\left(S_{i}, n_{i}\right)$ a pointed Riemann surface and $p_{i}: X_{i} \rightarrow\left(S_{i}, n_{i}\right)$ a covering projection, for $i=1,2$, and let $f:\left(S_{1}, n_{1}\right) \rightarrow\left(S_{2}, n_{2}\right)$ be a homeomorphism of pointed surfaces. If $X_{1}$ is simply connected and the degree or dihedral degree of $p_{1}$ is $n\left(p_{1}(z)\right)$ for $z \in X_{1}$, then for any interior point $x$ of $S_{1}$ such that $n(x)=1$ and for any pair of points

$$
z_{1} \in p_{1}^{-1}(x) \text { and } z_{2} \in p_{2}^{-1}(f(x))
$$

there is a unique mapping $f^{\prime}: X_{1} \rightarrow X_{2}$ such that the diagram

$$
\begin{array}{ccc}
X_{1} \stackrel{f^{\prime}}{\rightarrow} & X_{2} \\
p_{1} \downarrow & & \downarrow p_{2} \\
S_{1} \stackrel{f}{\rightarrow} & S_{2}
\end{array}
$$

commutes and $f^{\prime}\left(z_{1}\right)=z_{2}$.

Proof: We construct first $f^{\prime} \mid X_{1}^{\prime}$ when $X_{i}^{\prime}=p_{i}^{-1}\left(S_{i} \backslash|n|\right), \quad i=1,2$. Let $z \in X_{1}^{\prime}$; then there is a path $u: I \rightarrow X_{1}^{\prime}$ ( $I$ is the closed interval $[0,1])$ such that $u(1)=z$ and $u(0)=z_{1}$. Since the boundary of $S_{1}$ may be non-empty, we cannot define $f^{\prime}(z)=u^{\prime}(1)$ where $u^{\prime}: I \rightarrow X_{2}^{\prime}$ is a path such that 


$$
u^{\prime}(0)=z_{2} \text { and } p_{2} u=f p_{1} u
$$

for the path $u^{\prime}$ is not uniquely determined by these conditions. But if we note that by the definition of a covering projection there is a division $0=t_{0}<t_{1}<\ldots<t_{n}=1$ of $I$ and open sets $U_{i} \subset X_{1}^{\prime}$ and $U_{i}^{\prime} \subset X_{2}^{\prime}$ for $i=1, \ldots, n$, such that $u\left(\left[t_{i-1}, t_{i}\right]\right) \subset U_{i}$ and unique homeomorphism $f_{i}: U_{i} \rightarrow U_{i}^{\prime} \quad$ such that $p_{2} f_{i}=f\left(p_{1} \mid U_{i}\right), \quad f_{i} \mid U_{i} \cap U_{i-1}=$ $f_{i-1} \mid U_{i} \cap U_{i-1}$ and $f_{1}\left(z_{1}\right)=z_{2}, i \leq n$, we may set the additional condition

$$
u^{\prime} \mid\left[t_{i-1}, t_{i}\right]=f_{i}\left(u \mid\left[t_{i-1}, t_{i}\right]\right), \quad i \leq n,
$$

then $u^{\prime}$ is uniquely determined by $(* *)$ and satisfies besides (*). We set

$$
f^{\prime}(z)=u^{\prime}(1) \text {. }
$$

We must show that this does not depend on the choice of the path $u$. Since $X_{1}$ is simply connected it suffices to show that for paths of the form $u=v w v^{-1}$ where $v(0)=z_{1}$ and $w$ is a closed path such that

$$
w(t)=g\left(e^{2.7 i t} / 2\right)
$$

where $g: E \rightarrow U$ is a conformal equivalence from the open unit disk to the neighbourhood $U$ of a point $z^{\prime} \in X_{1} \backslash X_{1}^{\prime}$, the path $u^{\prime}$ corresponding to $u$ is closed, i.e. $u^{\prime}(0)=u^{\prime}(1)=z_{2}$. But this follows from the fact that the degree or dihedral degree of $p_{2}$ at a point $z^{\prime \prime} \in p_{2}^{-1}\left(f\left(p_{1}(z)\right)\right)$ divides the degree or dihedral degree of $p_{1}$ at $z^{\prime}$.

After that it is obvious how to extend the mapping $f^{\prime}$ to the discrete set $X_{1} \backslash X_{1}^{\prime}=p_{1}^{-1}(|n|)$.

Remark. The proposition 2.4 shows that $p_{1}: X_{1} \rightarrow\left(S_{1}, n_{1}\right)$ is, in a sense, a universal object in the category whose objects are covering projections $p: X \rightarrow\left(S_{1}, n_{1}\right)$. It can be shown that in the sense specified by the proposition 2.4, every pointed Riemann surface has a universal object $p: X \rightarrow(S, n)$, but, in general, the degree or dihedral degree of $p$ at points $z \in p^{-1}(x)$ may differ from $n(x), x \in S$. We do not pursue this subject further, for we are more interested in the corollaries of proposition 2.4 than in the proposition itself. Of these corollaries we prove first:

2.4.1 Corollary. Let $X=X_{1}, p=p_{1} . S=S_{1}, n=n_{1}$ be as above. Then the group $F$ of conformal equivalences of $X$ such that the triangle

$$
\underset{S \downarrow}{X \underset{S}{ } \underset{ }{f} X}
$$

commutes, is a discrete group of conformal mappings such that $\left(S_{F}, n_{F}\right)=$ $(S, n)$. If $(S, n)=\left(S_{G}, n_{G}\right)$ for some discrete group $G$ of $X$, then $F=G$. 
Proof: It suffices to take $X=X_{1}=X_{2}, p=p_{1}=p_{2}$ and $S=S_{1}=S_{2}$ in proposition 2.4. After that the proof is rather obvious.

2.4.2 Corollary. The solution of the problem 2.3 depends only on the homeomorphism class of the pointed Riemann surface $(S, n)$.

Proof: Let $\left(S_{i}, n_{i}\right)$ be two pointed Riemann surfaces. $i=1,2$, and let $f:\left(S_{1}, n_{1}\right) \rightarrow\left(S_{2}, n_{2}\right)$ be a homeomorphism of pointed surfaces. Suppose that $\left(S_{1}, n_{1}\right)=\left(S_{p}, n_{p}\right)$ for some covering projection $p: X \rightarrow\left(S_{1}, n_{1}\right)$ where $X$ is a simply connected Riemann surface and thus $\left(S_{1}, n_{1}\right)=$ $\left(S_{F}, n_{F}\right)$ by corollary 2.4 .1 for some discrete group $F$ of $X$. Then $f p: X \rightarrow\left(S_{2}, n_{2}\right)$ is not as a rule a covering projection of $\left(S_{2}, n_{2}\right)$ because it does not behave in the required manner with respect to the conformal structures. But it is not difficult to see that the conformal structure of $X$ may be redefined in such a way that $f p$ is a covering projection. Then the conclusion follows by corollary 2.4.1.

2.4.3 Corollary. Let $X_{1}$ and $X_{2}$ be two simply connected Riemann surfaces and $F_{i}$ a discrete group of $X_{i}, i=1,2$. Let $\left(S_{i}, n_{i}\right)=\left(S_{F_{i}}, n_{F_{i}}\right)$ for $i=1,2$, and let $f:\left(S_{1}, n_{1}\right) \rightarrow\left(S_{2}, n_{2}\right)$ be a homeomorphism of pointed surfaces. Let $f_{1}, f_{2}: X_{1} \rightarrow X_{2}$ be two homeomorphisms such that the diagram

$$
\begin{gathered}
X_{1} \stackrel{f_{i}}{\rightarrow} X_{2} \\
p_{1} \downarrow \underset{p_{2}}{ } \stackrel{\downarrow}{S_{1}} \underset{S_{2}}{\rightarrow} S^{2}
\end{gathered}
$$

commutes $(i=1,2)$ where $p_{1}$ and $p_{2}$ are the covering projections. Then

$$
f_{2}=S f_{1} \text { for some } S \in F_{2} \text {. }
$$

The mappings $f_{i}$ induce isomorphisms $\varphi_{i}: F_{1} \rightarrow F_{2}$ such that

$$
f_{i}(T(x))=q_{i}(T)\left(f_{i}(x)\right) \text { for } x \in X_{1}, T \in F_{1} \text {, and } i=1,2 .
$$

Then we have

$$
\varphi_{2}(T)=S \phi_{1}(T) S^{-1}
$$

where $S$ is the element of $F_{2}$ specified by (1) above.

This is again an obvious consequence of proposition 2.4.

It is clear that problem 2.3 does not always have a solution. Consider, for example, the case where $S=S^{2}$ is the one point compactification of the complex plane $\mathbf{C}, n \mid \mathbf{C} \equiv 1, n(\infty)>1$. Then $(S, n)$ cannot be represented in the form $\left(S_{F}, n_{F}\right)$ for any discrete group of conformal mappings of a simply connected Riemann surface $X$.

The case where $S$ is compact is well-known and leads to well-known groups of conformal mappings. (See e.g. Zieschang-Vogt-Coldewey [7]). 
Therefore we restrict our attention to the case of pointed surfaces $(S, n)$ where $S$ is non-compact and in this case show, disregarding the possible conformal structure of $(S, n)$, that it may be represented in the form $\left(S_{F}, n_{F}\right)$ where $F$ is a discrete group of conformal mappings of the unit disk. It will follow by corollary 2.4.2 that any pointed Riemann surface $(S, n), S$ non-compact, has a representation in the form $S_{F}, n_{F}$ ) where $F$ is a discrete group of the unit disk or the complex plane $\mathbf{C}$. The remaining sections of this chapter are devoted to this.

C. Discrete groups of conformal mappings of the unit disk. In this section we consider only groups containing no reflections. We first study some very simple discrete groups of the unit disk as well as their fundamental domains and introduce a principle of combining these groups into more complex groups of the unit disk. Later we show that every noncompact pointed surface without boundary can be represented in the form $\left(S_{F}, n_{F}\right)$ where $F$ is a group obtained this way.

Let $F$ be a cyclic group of conformal mappings of $E$. Since groups containing reflections have been excluded from the discussion the structure of $F$ depends on the order of $F$. We treat separately the cases where the order of $F$ is finite or infinite and agree on the conventions and notations to be used.

A. The order of $F$ is finite. $F$ is generated by a rotation $T$ through the angle $2 \pi / n, n \geq 2$, and has a fundamental domain $D_{T}$ which is closed in $E$ and whose sides are non-Euclidean rays $P_{T}$ and $N_{T}$ such that the intersection of $P_{T}$ and $N_{T}$ is the vertex $Q_{T}=\operatorname{Fix}(T)$ and such that

$$
T\left(N_{T}\right)=P_{T}
$$

We denote by $P_{T}^{\prime}$ the non-Euclidean line whose (ideal) endpoints on $S^{1}$ are the same as the (ideal) endpoints of $P_{T}$ and $N_{T}$. We denote by $D_{T}^{\prime}$ the component of $E \backslash P_{T}^{\prime}$ for which $D_{T}^{\prime} \cap\left(E \backslash D_{T}\right)=\varnothing$ and by $C_{T}^{\prime}$ the other component of $E \backslash P_{T}^{\prime}$.

If $n=2$, then $P_{T}^{\prime}=P_{T} \cup N_{T}$ and we say that $P_{T}^{\prime}$ is the side of $D_{T}$. In this case the fundamental domain has no vertices.

$B$. The order of $F$ is infinite. In this case $F$ is generated by a directly or indirectly conformal mapping $T$ and has a fundamental domain $D_{T}$, closed in $E$, whose sides are two non-intersecting non-Euclidean lines $P_{T}$ and $N_{T}$ such that

$$
T\left(N_{T}\right)=P_{T}
$$

In both cases $A$ and $B$ the complement of the fundamental domain $D_{T}$ in $E$ is denoted by

$$
C_{T}=E \backslash D_{T}
$$


Now we may state the principle of combination for groups of the form $A$ or $B$. Let $J$ be some index set, and suppose that for each $i \in J$ a group $F_{i}$ and its generator $T_{i}$ as well as a fundamental domain $D_{i}=D_{T_{i}}$ are given. We suppose that the triples $F_{i}, T_{i}, D_{i}, i \in J$, are as in $A$ or $B$. We shall use the notations introduced above in $A$ or $B$ replacing the double index $T_{i}$ by $i$, thus $P_{T_{i}}=P_{i}$ etc.

2.5 Definition. Let $F_{i}, D_{i}, C_{i}, T_{i}, i \in J$, be as above. If the inclusions

$$
\mathrm{cl}_{E} C_{i} \subset \operatorname{int}_{E} D_{j} \text { for } i, j \in J, i \neq j,
$$

are valid in (1), then we say that the system $F_{i}, D_{i}, T_{i}, J$ forms a free combination of cyclic groups.

We shall find it later important to be able to decide whether a given system $F_{i}, D_{i}, T_{i}, J$ forms a free combination of cyclic groups by considering inclusions of the form

$$
\operatorname{cl}\left(C_{i}\right) \cap S^{1} \subset \operatorname{cl}\left(D_{j}\right) \cap S^{1} \text { for } i, j \in J, i \neq j .
$$

This condition is at first appearance not equivalent with (1). It is clear that the inclusions (2) are implied by (1). On the other hand the inclusions (2) almost imply the inclusions (1). The only case when this is not true is the case when $J$ consists of two elements, say $J=\{1,2\}, T_{i}$ is elliptic of order two for $i=1,2$ int $_{E} D_{1}=C_{2}$ and $\operatorname{int}_{E} D_{2}=C_{1}$. Taking account of this exceptional case we may express the definition 2.5 in the equivalent form:

2.5' Definition. Let $F_{i}, D_{i}, T_{i}, J$ be as above. Then they form a free combination of cyclic groups if the inclusions (2) are satisfied and if in addition it is proper when $T_{i}$ and $T_{j}$ are elliptic of order two.

Before stating the main theorem of this section we introduce the following notation. Let $F$ be a group of conformal mappings of the unit disk. Then we set

$$
\operatorname{Fix}(F)=\{P(T): T \in F, T \text { has infinite order }\},
$$

which is a subset of $S^{1}$.

2.6. Theorem. Let $F_{i}, D_{i}, T_{i}, J$ form a free combination of cyclic groups and let $F$ be the group generated by $F_{i}, i \in J$. Then $F$ is the free product of the groups $F_{i}, i \in J$. Moreover, if one of the following conditions is true

(a) $\operatorname{Fix}(F)$ is dense in $S^{\mathbf{1}}$,

(b) $N_{i}$ is an arc of the isometric circle of $T_{i}, i \in J$,

then $F$ is a discrete group of conformal mappings of the unit disk with a fundamental domain

$$
D=\bigcap_{i \in J} D_{i} .
$$


In the case $(b)$, the set $\operatorname{Fix}(F)$ is dense in $S^{1}$ if and only if $\operatorname{cl} D \cap S^{1}$ contains no intervals of $S^{1}$.

Proof: We first prove the followirg assertion where we call a nonEuclidean line or ray a side of $D$ if it is a side of $D_{i}$ for some $i \in J$ and a point a vertex of $D$ if it is a vertex for $s$ me $D_{i}, i \in J$.

A. Let $T=T_{1} \ldots T_{n}$ be an element of $F$ such that $T_{k} \in F_{i_{k}} \backslash\{1\}$, $1 \leq k \leq n$, and $i_{k} \neq i_{k-1}$ for $k=2, \ldots, n$. If the order of $F_{i_{k}}$ is infinite let $C_{i_{k}}^{\prime}$ be the component of $C_{i_{k}}$ that contains $T_{k}\left(\operatorname{int}_{E} D_{k}\right)$, otherwise let $C_{i_{k}}^{\prime}=C_{i_{k}}$. Then

(a) $T(D) \subset \mathrm{cl}_{E} \mathrm{C}_{i_{\mathrm{i}}}^{\prime}$

(b) $T(D) \cap D=\varnothing$ if $n>1$ or if $n=1$ and the order of $F_{i_{1}}$ is infinite and $T_{1}$ is not a generator of $F_{i_{1}}$,

$=a$ vertex of $D$ if $n=1$ and the order of $F_{i_{1}}$ is finite with $T_{1}=T_{i_{1}}^{k}$ where $1<k<\left(\right.$ the order of $\left.F_{i_{1}}\right)-1$, $=a$ side of $D$ if $n=1$ and $T_{1}=T_{i_{1}}$ or $T_{1}=T_{i_{1}}^{-1}$.

We prove $A$ by induction on $n$. The case $n=1$ is clear. If $A$ has already been proved for $T^{\prime}=T_{2} \ldots T_{n}$, then

$$
T^{\prime}(D) \subset \mathrm{cl}_{E} C_{i_{2}}^{\prime} \subset \operatorname{int}_{E} D_{i_{1}}
$$

From this follows the conclusion.

It is a consequence of $A$ that $F$ is the free product of the groups $F_{i}, i \in I$, and that $D$ is a fundamental domain of the discrete group $F$ if we can show that

$$
D^{\prime}=\bigcup_{T \in F} T(D)
$$

is the whole open unit disk.

To prove this we set

$$
\mathbf{D}=\{T(D): T \in F\}
$$

and introduce the notion of a chain in D. A collection $\left\{D_{n}: n \geq 0\right\}$ is called a chain of $\mathbf{D}$ if

(i) $D_{n} \cap D_{n+1}$ is a common side or a common vertex, $n \geq 0$,

(ii) $D_{n-1}$ and $D_{n+1}$ are in different components of $E \backslash$ (int $D_{n}$ ), $n \geq 1$. Besides we make the following assumption which is quite unessential but will simplify notations:

(iii) $D_{0}=D$.

Then it is easy to see that there is a unique sequence of elements of $F T_{1}$, $T_{2}, \ldots$ such that

$$
D_{n}=T_{1} \ldots T_{n}(D)
$$

where each $T_{n} \in F_{i_{n}} \backslash\{1\}$ for some $i_{n} \in J$ and satisfies 
(a) If $F_{i_{n}}$ is infinite cyclic, then $T_{n}$ is a generator of $F_{i_{n}}$, and if $i_{n+1}=i_{n}$, then $T_{n+1}$ is $T_{n}, n \geq 1$,

(b) If $F_{i_{n}}$ is finite, then $i_{n+1} \neq i_{n}$.

Correspondingly, given a sequence $T_{n} \in F_{i_{n}} \backslash\{1\}$ and satisfying the conditions (a) and (b) above, the domains $D_{0}=D$ and $D_{n}=T_{1} \ldots T_{n}(D)$ form a chain of $\mathbf{D}$.

Given a chain $\left\{D_{n}\right\}$ of $\mathbf{D}$ we define a sequence $K_{1}, K_{2}, \ldots$ of nonEuclidean lines by

$$
\begin{aligned}
K_{n}= & D_{n-1} \cap D_{n} \text { if } F_{i_{n}} \text { is infinite cyclic }(i \geq 1), \\
K_{n}= & \left.T_{1} \ldots T_{n-1}\left(P_{i_{n}}^{\prime}\right) \text { if } F_{i_{n}} \text { is finite (if } F_{i_{1}} \text { is finite } K_{1}=P_{i_{1}}^{\prime}\right) \\
& (n \geq 1) .
\end{aligned}
$$

Then it is easily verified, using the properties (i) and (ii) of a chain of $\mathbf{D}$, that the $K_{n}, n \geq 1$, form a collection of disjoint non-Euclidean lines such that $K_{n+1}$ and $K_{n+2}$ are in the same component $Q_{n}$ of $E \backslash K_{n}$. Hence the lines $K_{m}, m \geq n$, are in the component $Q_{n}$ of $E \backslash K_{n}$. (Incidentally, we now see that all the elements of a chain $\left\{D_{n}\right\}$ of $\mathbf{D}$ must be different.) The intersection

$$
Q=\bigcap_{n \geq 1} \operatorname{cl} Q_{n}
$$

is either a point of $S^{1}$ or of the form $K \cap E^{1}$ where $K$ is a closed disk whose boundary is orthogonal to the unit circle. It is easy to see:

B. If $\left\{D_{n}\right\}$ is a chain of $\mathbf{D}$, then the intersection of $Q$ with $D^{\prime}$ in (1) is empty.

For let $D_{n}=T_{1} \ldots T_{n}(D), n \geq 0$, be a chain of $\mathbf{D}$ where the elements $T_{n} \in F$ are as above. Let $C=T(D)$ be an arbitrary element of $\mathbf{D}$ where $T \in F$ may be written in the form $T=T_{1}^{\prime} \ldots T_{k}^{\prime}$ where $T_{n}^{\prime}, 1 \leq n \leq k$, satisfy (a) and (b) modified to the finite case. If $T_{n}^{\prime}=T_{n}$ for $n \leq m$ and $T_{m+1}^{\prime} \neq T_{m+1}$ where $m<k$, then $C \cap \operatorname{cl} Q_{n}=\varnothing$ for $n>m$. Otherwise $C \cap \mathrm{cl} Q_{n}=\varnothing$ for $n>k+1$.

We associate with each point $x \in E \backslash D^{\prime}$ a chain of $\mathbf{D}$ such that:

(i) $D_{0}=D$.

(ii) Let $D_{n}$ be the unique element of $\mathbf{D}$ such that $D_{n} \cap D_{n-1}$ is a common side or common vertex and that $x$ and $D_{n-1}$ are in different components of $E \backslash\left(\right.$ int $\left.D_{n}\right)$. (This implies that $D_{n+1}$ and $D_{n-1}$ are in different components of $\left.E \backslash\left(\operatorname{int} D_{n}\right)\right)$.

Then $x \in Q$ where $Q$ is defined as in (4) by means of the chain $\left\{D_{n}\right\}$. In this case $Q$ cannot be a point since it contains a point of $E$. We now derive a contradiction from this if one of the assumptions (a) or (b) of theorem 2.6 is true. 
Under the assumption (a) the set $\operatorname{Fix}(F)$ is dense in $S^{1}$. In particular there is an element $T \in F$ such that $P(T) \in \operatorname{int}_{S^{1}} Q \cap S^{1}$ which is an open interval of $S^{1}$. But this contradicts assertion $B$, since $Q \cap D^{\prime}=\varnothing$ and in every neighbourhood of $P(T)$ there are transforms $T^{n}(z)$ of an arbitrary point $z$ of $E$.

The situation is somewhat more complicated under the assumption (b) of theorem 2.6. We prove that given $d>0$, there is an integer $n_{0}$ such that

$$
\operatorname{diam}\left(K_{n}\right)<d \text { for } n>n_{0} .
$$

where $K_{n}$ is the sequence of open arcs of circles orthogonal to $S^{1}$ defined by equation (3) and arrive in this way to a contradiction with the assumption $D^{\prime} \neq E$. Let

$$
D_{n}=T_{1} \ldots T_{n}(D)
$$

where $T_{n} \in F_{i_{n}} \backslash\{1\}$ satisfy conditions (a) and (b). Then

$$
K_{n}=T_{1} \ldots T_{n-1}\left(K_{n}^{\prime}\right)\left(=K_{1}^{\prime} \text { if } n=1\right), n \geq 0,
$$

where $K_{n}^{\prime}$ is a side of $D$ or the non-Euclidean line $P_{i_{n}}^{\prime}$ if $F_{i_{n}}$ is finite. It follows easily by A that $T_{m} \ldots T_{n-1}\left(K_{i}^{\prime}\right)$ is outside the isometric circle of $T_{m-1}$ and thus we have the increasing sequence

$\operatorname{diam}\left(K_{n}\right)=\operatorname{diam} T_{1} \ldots T_{n-1}\left(K_{n}^{\prime}\right)<\operatorname{diam} T_{2} \ldots T_{n-1}\left(K_{n}^{\prime}\right)<\ldots<\operatorname{diam}\left(K_{n}^{\prime}\right)$.

Since by A $T_{m} \ldots T_{n-1}\left(K_{n}^{\prime}\right) \subset C_{i_{m}}$ we may draw the conclusion that the diameter of $K_{n}$ does not exceed the diameter of the components $C_{i_{m}}$, $m<n$. Thus if $\operatorname{diam}\left(K_{n}\right)<d$ for all $n>0$, then in the sequence $T_{1}$, $T_{2}, \ldots$ only a finite number of elements can occur, i.e. those elements $T_{n} \in F_{i_{n}}$ for which the diameter of components of $C_{i_{n}}$ exceeds $d$. We denote this finite set of elements of $F_{i_{n}} \backslash\{1\}, n>1$, by $X$.

We are now in a position to use proposition 1.3. In this connexion we must remember that among the transformations of $X$ there may be some whose isometric circle is not unique, i.e. the elements of an elliptic cyclic subgroup whose fixed point is the origin. We denote this subgroup by $F_{0}$ if it exists. Then, by proposition 1.3 , there is a real $k, 0<k<1$, such that

$$
\operatorname{diam}\left(K_{n}\right) \leq k^{j} \operatorname{diam}\left(K_{n}^{\prime}\right)
$$

where $j$ is the number of elements in the sequence $T_{1}, \ldots, T_{n-1}$ that do not belong to $F_{0}$. Since $j \rightarrow \infty$ when $n \rightarrow \infty$ a contradiction can be derived from this if it is assumed that $\operatorname{diam}\left(K_{n}\right)>d$ for all $n$.

It remains to prove that in case (b) the set $\operatorname{Fix}(F)$ is dense in $S^{1}$ whenever cl $D \cap S^{1}$ does not contain intervals of $S^{1}$. Since, evidently, $\operatorname{Fix}(F)$ 
cannot be dense in $S^{1}$ if $\operatorname{cl} D \cap S^{1}$ contains intervals of $S^{1}$, we must only prove the converse assertion. In this case $J$ contains at least two elements. We have:

C. If the index set $J$ contains two indexes, then for each component $C$ of $C_{j}, j \in J$, there is a transformation $T \in F$ of infinite order such that $P(T) \in \mathrm{cl} C$.

To prove $C$ we choose another $i \in J, i \neq j$. Let $T \in F_{j}$ be an element such that $T\left(D_{j}\right) \subset \mathrm{cl} C$. Then

$$
T T_{i}(C) \subset T\left(C_{i}\right) \subset T\left(D_{j}\right) \subset \mathrm{cl} C
$$

and thus $P\left(T T_{i}\right) \in \operatorname{cl} C \cap S^{1}$.

Now let $x$ be an arbitrary point of $S^{1}$. If there is $T \in F$ such that every neighbourhood of $x$ contains components of $E \backslash T(D)$ conclusion follows by $C$. If this is not the case there is a chain $\left\{D_{n}\right\}$ of $\mathbf{D}$ such that:

(i) $D_{0}=D$,

(ii) $D_{n}$ is an element of $\mathbf{D}$ such that $D_{n} \cap D_{n-1}$ is a common side or a common rertex and that $D_{n}$ and $D_{n-2}$ are in different components of $E \backslash$ (int $\left.D_{n-1}\right)(n \geq 2)$ and that there is a component $C$ of $E \backslash D_{n}$ such that $x \in \operatorname{cl} C, D_{n-1} \cap C=$.

It is quite evident that there is such a chain and that if $Q_{i}, i \geq 1$. is defined as above corresponding to the chain $\left\{D_{n}\right\}$ then $x \in Q_{i}$ for all $i \geq 1$, and thus

$$
x \in Q=\bigcap_{i \geq 1} \operatorname{cl} Q_{i}
$$

We have shown that $\operatorname{diam}\left(\operatorname{cl} Q_{i}\right) \rightarrow 0$ as $i \rightarrow \infty$. Since every $Q_{i}$ contains transforms of $D$ (e.g. $D_{n}$ for large enough $n$ ) the conclusion follows by $C$.

D. Standard representations for non-compact pointed surfaces without boundary. It is well-known that every compact surface can be obtained from the closed unit disk $E^{1}$ by identifying suitably intervals of $S^{1}$. We prove here a similar representation theorem for non-compact pointed surfaces without boundary.

Let $(S, n)$ be a non-compact pointed surface without boundary. Let

$$
E^{\prime}=E \cup\left(\bigcup_{i \in K^{\prime}} I_{i}\right)
$$

where each $I_{i}, i \in K$, is an open or half-open interval of $S^{1}$ such that

$$
I_{i} \cap I_{j}=\varnothing \quad \text { or }
$$

a common endpoint for $i, j \in K, i \neq j$.

Further, we set 


$$
V=\left\{Q: Q \in I_{i} \cap I_{J}, i, j \in K, i \neq j\right\}
$$

and let $i: E^{\prime} \cap S^{1} \rightarrow E^{\prime} \cap S^{1}$ be a homeomorphism such that

$$
\begin{aligned}
i^{2} & =i d, i\left(I_{i}\right)=I_{j} \text { where } i \neq j, i, j \in K \text {, and } \\
i \backslash V & =i d .
\end{aligned}
$$

Then if we identify $x$ with $i(x)$ for $x \in E^{\prime} \cap S^{1}$ we obtain a surface denoted by $S^{\prime}$; let $k: E^{\prime} \rightarrow S^{\prime}$ be the canonical surjection. If there is a homeomorphism $f: S^{\prime} \rightarrow S$ such that

$$
|n|=f(k(V))
$$

we say that $R=\left(E^{\prime}, i, f\right)=\left(E^{\prime}, i, k, S^{\prime}, f\right)$ is a standard representation of $(S, n)$.

We may note that the set of intervals $I_{i}, i \in K$, is uniquely determined by the mapping $i$. The set $V$ defined by (1) is also defined by

$$
V=\left\{x \in E^{\prime} \cap S^{1}: i(x)=x\right\}
$$

and is called the set of vertices of the representation $R$.

The remainder of this section is devoted to proving the following proposition:

2.7 Proposition. Let $(S, n)$ be a pointed surface without boundary such that $S$ is non-compact and connected. Then $(S, n)$ has a standard representation $\left(E^{\prime}, i, f\right)$. If $(S, n)$ is not topologically equivalent with $(E$. 1) then we may suppose in addition:

(i) The set $S^{1} \backslash E$ does not contain intervals of $S^{1}$.

(ii) Let $V$ be the set of vertices of $\left(E^{\prime}, i, f\right)$ (i.e. the set of fixed points of $i)$. Then it can be supposed that $\left(E^{\prime} \cap S^{1}\right) \backslash V$ is a union of open intervals such that they are identified in pairs by $i$ and any two intervals identified by $i$ have equal length.

The proof is based on the fact that every non-compact connected surface with countable basis has a canonical exhaustion, i.e. there is a sequence $K_{1} \subset K_{2} \subset \ldots$ of compact bordered subsurfaces of $S$ such that

(i) $\bigcup_{i=1}^{\infty} K_{i}=S$,

(ii) $K_{i} \subset \operatorname{int} K_{i+1}$ for $i \geq 1$,

(iii) Each component of $\operatorname{cl}\left(K_{i+1} \backslash K_{i}\right)$ is a connected subsurface of $S$ having in common with $K_{i}$ exactly one boundary component.

(iv) Each component of $\operatorname{cl}\left(S \backslash K_{i}\right)$ is non-compact, $i \geq 1$.

It follows from these properties that each $K_{i}$ is connected. A proof of the existence of such an exhaustion of $S$ can be found in Ahlfors-Sario [1]. Besides the above conditions we can obviously demand 
(v) $n \mid \cap K_{i} \subset \operatorname{int} K_{i}$ for $i \geq 1$.

A canonical exhaustion of $S$ that satisfies the condition $(v)$ is said to be a canonical exhaustion of the pointed surface $(S, n)$.

In what follows it is quite essential to have a convenient representation for compact bordered pointed surfaces $(K, m)$ such that $|m|$ does not contain boundary points of $K$. The representation we use is quite similar to the standard representation for open pointed surfaces without boundary given above.

Let $I_{i}, i \in L$, be closed arcs of circles orthogonal to $S^{1}$, lying in $E^{1}$, such that the endpoints of each $I_{i}, i \in L$, are on $S^{1}$. We suppose that this set is a disjoint set of circle ares. Besides we suppose that a set $I_{i}$, $i \in K$, of closed intervals of $S^{1}$ is given such that at least one endpoint of $I_{i}$ is an endpoint of some $I_{k}, k \in L$. The other endpoint is either an endpoint for another $I_{l}, l \in L$ or an endpoint for $I_{j}, j \in K$. Except for the endpoints the sets $I_{i}, i \in K$, form a disjoint family. Further, we require that

$$
A=\left(\bigcup_{i \in K} I_{i}\right) \cup\left(\bigcup_{i \in L} I_{i}\right)
$$

is a Jordan curve bounding a Jordan region $E^{\prime}$ of $E^{1}$. We suppose that $E^{\prime}$ is closed, i.e. $A \subset E^{\prime}$. The set $V \subset \bigcup_{i \in K} I_{i}$, called the set of vertices of the representation, is defined exactly as above by the equation (1). In the same way, we suppose that a homeomorphism $i: E^{\prime} \cap S^{1} \rightarrow E^{\prime} \cap S^{1}$ is given satisfying the equations (2) above. Then if the points $x$ and $i(x)$ are identified, $x \in E^{\prime} \cap S^{1}$, we obtain a bordered surface, denoted by $S^{\prime}$, with the canonical projection $k: E^{\prime} \rightarrow S^{\prime}$. Then if there is a homeomorphism $f: S^{\prime} \rightarrow K$ such that $m=f(k(V))$, we say that $\left(E^{\prime}, i, f\right)$ is a representation for $(K, m)$. It may also be denoted by

$$
R=\left(E^{\prime}, i, f\right)=\left(E^{\prime} . i, f \cdot S^{\prime}, k\right)
$$

if it is desirable to choose a notation for the factor space $S^{\prime}$ and the canonical surjection $k$.

Let $R_{j}=\left(E_{j}, i_{j}, f_{j}, S_{j}^{\prime}, k_{j}\right)$ be representations for two compact, bordered surfaces $\left(K_{j}, n_{j}\right), j=1,2$, such that $K_{1}$ is a subsurface of $K_{2}$ by which we mean that $K_{1} \subset K_{2}$ and that each boundary component of $K_{1}$ is either a boundary component of $K_{2}$ or belongs to the interior of $K_{2}$. Further, suppose that $E_{1}^{\prime} \subset E_{2}^{\prime}$ and that $i_{2} E_{1}^{\prime} \cap S^{1}=i_{1}$. Then we may regard $S_{1}^{\prime}$ as a subsurface of $S_{2}^{\prime}$ and if in addition

$$
f_{2}^{\prime} \mid S_{1}^{\prime}=f_{1}^{\prime}
$$

we say that $R_{2}$ is an extension of $R_{1}$, written $R_{1} \subset R_{2}$. 
We suppose that a canonical exhaustion $K_{1} \subset K_{2} \subset \ldots$ of a noncompact, connected pointed surface $(S, n)$ without boundary is given. We exclude the trivial case where $S$ is homeomorphic to the open unit disk and $n \equiv 1$. Then we may assume that the pointed surface $\left(K_{1}, n \mid K_{1}\right)$ is not topologically equivalent to the pair $\left(E^{1}, 1\right)$ where 1 denotes the function $E^{1} \rightarrow \mathrm{N}$ having the constant value 1. Then, using the well-known classification theorems for compact, bordered surfaces, (see e.g. AhlforsSario [1]), it is easy to see that $\left(K_{1}, n \mid K_{1}\right)$ has a standard representation $R_{1}=\left(E_{1}^{\prime}, i_{1}, f_{1}, S_{1}^{\prime}, k_{1}\right)$. We show that this can be extended to a representation $R_{2}$ of $\left(K_{2}, n \mid K_{2}\right)$. Let $K$ be component of $\operatorname{cl}\left(K_{2} \backslash K_{1}\right)$. Then $K$ is a compact bordered surface having at least two boundary components of which exactly one, say $B$, is in common with $K_{1}$, i.e.

$$
B=K \cap K_{1} .
$$

It is easy to see that the pointed surface $(K, n \mid K)$ has a representation $R=\left(E^{\prime}, i, f, S^{\prime}, k\right)$ such that $A=E_{1}^{\prime} \cap E^{\prime}$ is an arc of a circle orthogonal to $S^{1}$ and that

$$
B=f(k(A)) \text {. }
$$

Let $A, O_{1}, \ldots, O_{n}$ be the disjoint set of circle ares orthogonal to $S^{\mathbf{1}}$ such that

$$
B=f_{1}\left(k_{1}\left(A \cup O_{1} \cup \ldots \cup O_{n}\right)\right)
$$

(We may suppose that $A \subset f_{1}^{-1}\left(k_{1}^{-1}(B)\right)$ ). We choose the arcs of circles $O_{1}^{\prime} \ldots, O_{n}^{\prime}$ orthogonal to $S^{1}$ such that $O_{i}^{\prime}$ and $E_{1}^{\prime} \backslash O_{i}$ are in different romponents of $E^{1} \backslash O_{i}, i \leq n$. Let $G_{i}$ be the region of $E$ whose boundary (in $E$ ) is $O_{i} \cup O_{i}^{\prime}, i \leq n$. Define

$$
E^{\prime \prime}=E_{1}^{\prime} \cup E^{\prime} \cup \operatorname{cl}\left(G_{1} \cup \ldots \cup G_{n}\right)
$$

Then it is easy to see that there is a representation $R^{\prime \prime}=\left(E^{\prime \prime}, i^{\prime \prime}, f^{\prime \prime}, S^{\prime \prime}, k^{\prime \prime}\right)$ of $K_{1} \cup K$ that is an extension of $R_{1}$. (Note that if $B \neq f_{1} k_{1}(A)$ ) it is not an extension of $R ; K \backslash f^{\prime \prime}\left(k^{\prime \prime}\left(E^{\prime}\right)\right)$ is homeomorphic to $[0,1] \times(0,1)$.) Treating separately each component of $\operatorname{cl}\left(K_{2} \backslash K_{1}\right)$ we obtain a representation $R_{2}$ that is an extension of $R_{1}$.

By now it is clear how to continue. We extend inductively the representation $R_{k-1}$ of $\left(K_{k-1}, n \mid K_{k-1}\right)$ to $\left(K_{k}, n \mid K_{k}\right)$ and obtain a sequence $R_{1} \subset R_{2} \subset \ldots$ of representations of $\left(K_{1}, n \mid K_{1}\right) \subset \ldots$. It may be supposed that at each stage of construction the orthogonal circle arcs bounding $E_{n}^{\prime}$ are arbitrarily small when $R_{n}=\left(E_{n}^{\prime}, i_{n}, f_{n}\right)$, e.g. we may demand that if $C$ is a component of $E^{1} \backslash E_{n}^{\prime}$ then

$$
\operatorname{diam} C<1 / n \text {. }
$$


After that it is quite obvious how to define the desired standard representation of $(S, n)$. Set

$$
E^{\prime}=\bigcup_{i=1}^{\infty} E_{i}^{\prime}
$$

Then, by (4), $E \subset E^{\prime}$ and we may note that $S^{1} \cap E^{\prime}$ has the property:

(a) $S^{1} \cap E^{\prime}$ does not contain intervals of $S^{1}$.

The mapping $i: E^{\prime} \cap S^{1} \rightarrow E^{\prime} \cap S^{1}$ is defined by the formula:

$$
i \mid E_{n}^{\prime} \cap S^{1}=i_{n} \text {. }
$$

Similarly, the homeomorphism $f: S^{\prime} \rightarrow S$ is defined by the formula:

$$
f \mid S_{n}^{\prime}=f_{n} \text {. }
$$

The verification that $\left(E^{\prime}, i, f, S^{\prime}, k\right)$ is a standard representation of $(S, n)$ is trivial. Besides it satisfies the condition (i) of proposition 2.7. It is possible that it does not have the property (ii), but by investigating how the representation of $\left(K_{1}, n \mid K_{1}\right)$ was extended to a representation of $\left(K_{2}\right.$, $\left.n \mid K_{2}\right)$ we may observe that the intervals of $\left(S^{1} \cap E^{\prime}\right) \backslash V$ (where $V$ is the set of vertices of the representation) may be indexed in such a way that we have a sequence $I_{1}, I_{2}, \ldots$ of intervals of $\left(E^{\prime} \cap S^{1}\right) \backslash V$ and that there is a sequence $n_{1}<n_{2}<$. of integers such that

(b) If $k, l>n_{i}>k^{\prime}, l^{\prime}$ and the intervals $I_{k}$ and $I_{l}$ are identified $b y$ $i$, then $I_{k}$ and $I_{l}$ are in the same component of $S^{1} \backslash\left(I_{k} \cup I_{l}\right)$. $\left(k, l, k^{\prime}, l^{\prime} \in \mathbf{N}\right)$.

Then if the standard representation $\left(E^{\prime}, i, f\right)$ does not have the property (ii), it can be reconstructed by (b) in such a way that it has both properties (i) and (ii).

E. The representation of non-compact pointed surfaces by means of discrete groups of the unit disk. In this section we give an affirmative answer to problem 2.3 in case of a non-compact pointed surface. The following theorem is based essentially on theorem 2.6 and proposition 2.7. If the boundary of $S$ is non-empty, use is made also of corollary 2.4.1.

Before we state the next theorem we agree on some notations conceming pointed surfaces with non-empty boundary. Let $(S, n)$ be a connected pointed surface whose boundary is non-empty. Its double $\left(S^{*}, n^{*}\right)$ is defined as follows: Symbolically, we denote

$$
S^{*}=S\lfloor S / \partial S
$$

where $\partial S$ is the boundary of $S$. This means that we take two copies of 
the surface $S$, form their disjoint union, and identify the corresponding boundary points. Thus $S^{*}$ is a connected surface without boundary. There is a canonical projection

$$
p^{*}: S^{*} \rightarrow S
$$

and two inclusions $i_{j}: S \rightarrow S^{*}, j=1,2$. Since $i_{1} \partial S=i_{2} \partial S$, the boundary of $S$ may be imbedded uniquely in $S^{*}$. Thus we may agree that $\partial S$ is a subset of $S^{*}$. Besides the above mentioned mappings we have still an involution of $S^{*}$, denoted $P$, that might be called a reflection about $\partial S$, such that

$$
i_{2}=P i_{1}
$$

After that we define a mapping $n^{*}: S^{*} \rightarrow \mathbf{N}$ such that $n^{*}(x)=$ $n\left(p^{*}(x)\right)$. The support of $n^{*}$ is discrete and therefore we have a connected pointed surface $\left(S^{*}, n^{*}\right)$.

We may now state the main theorem of this section:

2.8 Theorem. Let $(S, n)$ be a non-compact connected pointed surface. Then up to a homeomorphism

$$
(S, n)=\left(S_{F}, n_{F}\right)
$$

where $F$ is some discrete group of conformal mappings of the unit disk. Besides we have:

(i) If the boundary of $S$ is empty, $F$ can be chosen to be a free combination of cyclic groups and is thus a free product of cyclic groups.

(ii) The set $\operatorname{Fix}(F)=\{P(T): T \in F, T$ has infinite order $\}$ may be supposed to be dense in $S^{1}$ provided $S$ has no boundary and is not topologically equivalent with one of the following cases:

(a) $(E, m)$ where $m$ contains at most one point. Then $F$ is a finite cyclic group or $F=\{i d\}$.

(b) A spehere punctured at two points or a projective plane purctured at one point, with $n=\varnothing . F$ is an infinite cyclic group.

(c) $(E, m)$ where $m$ consists of tuo points and $y$ such that $m(x)$ $=m(y)=2 . F$ is the free product of two groups isomorphic with $\mathbf{Z}_{2}$.

(iii) If $F$ is a discrete group of conformal mappings of the unit disk, then $\left(S_{F}, n_{F}\right)$ (or $\left(S_{F}^{*}, n_{F}^{*}\right)$ if $S_{F}$ has boundary components) is topologically equivalent to one of the pointed surfaces in $(a),(b)$ or $(c)$ if and only if $F$ does not contain a free subgroup with two generators. If this is the case, $\operatorname{Fix}(F)$ contains at most two points.

Proof: We first consider the case where the boundary of $S$ is empty. If $(S, n)$ is topologically equivalent to $(E, 1)$, then we can obviously set $F=\{i d\}$. In all other cases we can choose a standard representation $\left(E^{\prime}, i, f\right)$ for $(S, n)$ satisfying conditions (i) and (ii) of proposition 2.7. 
If $E^{\prime} \cap S^{1}$ is an interval, we have the case (a) where $|n|$ contains exactly one point. Then, evidently, $(S, n)$ is topologically equivalent to $\left(S_{F}, n_{F}\right)$ where $F$ is a rotation group, thus a finite cyclic group. If $E^{\prime} \cap S^{\mathbf{1}}$ consists of two intervals and $n \equiv 1$, we have the case $(b)$. Then $(S, n)$ is topologically equivalent to $\left(S_{F}, n_{F}\right)$ where $F$ is the infinite cyclic group generated by a hyperbolic transformation $T$ of the unit disk, that may be, in the terminology we have adopted, either directly or indirectly conformal.

The case (c) deserves a special treatment. We choose two points $x, y \in E$ and denote by $T$, resp. by $S$, a rotation of the unit disk through the angle $\pi$ having the fixed point $x$, resp. $y$. We suppose that $x \neq y$, i.e. $S \neq T$. Then $\mathrm{ST}$ is a hyperbolic transformation whose axis passes through $x$ and $y$ and is uniquely determined by these. It is easy to see that every element of infinite order in the group $F$ generated by $S$ and $T$ is of the form $(S T)^{n}, n \in \mathbf{Z}, n \neq 0$. Every other element of $F$, different from 1, may be written in the form $(S T)^{n} X(S T)^{-n}$ where $X$ is $S, T, T S T$ or $S T S$. It is a rotation of order two such that its fixed point lies on the axis of $S T$. The pointed surface $(S, n)$ is homeomorphic to $\left(S_{F}, n_{F}\right)$.

After that we treat the general case, i.e. the case of an open connected pointed surface without boundary that is not equivalent to the surfaces in (a), (b) or (c). In this case $E^{\prime} \cap S^{1}$ contains at least two open intervals. We denote these by $I_{k}, k \in K$. The index set $K$ may be represented as a disjoint union

$$
K=K_{1} \cup K_{2} \cup K_{3}
$$

such that $i\left(I_{k}\right)=I_{l}$ for $k \in K_{1}$ and where $l \in K_{2}$. For $k \in K_{3}$ we have $i\left(I_{k}\right)=I_{k}$. We choose some $k \in K_{1}$. Let $O_{k}$ be the circle orthogonal to $S^{1}$ that passes through the endpoints of $I_{k}$. Similarly for $k \in K_{2}$. Then by proposition 1.1 there is a directly or indirectly conformal mapping $T_{k}$ of the unit disk such that the isometric circle of $T_{k}$ is $O_{k}$ and that of $T_{k}^{-1}$ is $O_{l}$ where $i\left(I_{k}\right)=I_{l}$ and such that $T_{k}\left(O_{k}\right)=O_{l}$. Let $T_{k}$ be indirectly conformal if $i$ preserves orientation on $I_{k}$, otherwise directly conformal. Let then $k$ be an index of $K_{3}$. Let $n_{k}=n\left(f\left(k\left(x_{k}\right)\right)\right)$ where $k$ is the canonical surjection of the representation $\left(E^{\prime}, i, f\right)=\left(E^{\prime}, i, f\right.$, $\left.S^{\prime}, k\right)$ and $x_{k}$ is the vertice of this representation contained in $I_{k}$. Let $I_{k}$ be the interval $I$ of the proposition 1.2 and let $O_{k}^{i}$ be the circle $O_{i}$ in the same proposition, $i=1,2$. Finally, let $T_{k}$ be the transformation $T$ of proposition 1.2. It may happen that the circles $O_{k}^{i}$ are lines passing through the origin. Then, in the following, by the region of $E$ outside the circles $O_{k}^{1}$ and $O_{k}^{2}$ we mean the component of $E \backslash\left(O_{k}^{1} \cup O_{k}^{2}\right)$ whose intersection with other circles $O_{l}, l \in K_{1} \cup K_{2}$ and $O_{l}^{1}$ and $O_{l}^{2}, \quad l \in K_{3}$, is non-empty.

Let $D_{k}$ be the closed domain of $E$ whose boundaries are the arcs 
$O_{k} \cap E$ and $O_{l} \cap E$, for $k \in K_{1}$ and $i\left(I_{k}\right)=I_{l}$. For $k \in K_{3}$, let $D_{k}$ be the closure of the region outside the circles $O_{k}^{1}$ and $O_{k}^{2}$.

We claim that the system $T_{k}, D_{k}, K_{1} \cup K_{3}$ is a free combination of cyclic groups and that the group $F$ generated by $T_{k}, k \in K_{1} \cup K_{3}$ is a discrete group of the unit disk whose fundamental domain is $D=\bigcap_{k \in K_{1} \cup K_{3}} D_{k}$. These are immediate consequences of the definition $2.5^{\prime}$ and proposition 2.6. Besides it can be seen that $\operatorname{Fix}(F)$ is dense in $S^{1}$ since cl $D \cap S^{1}$ does not contain intervals of $S^{1}$. This is again a consequence of proposition 2.6. Finally, it is easily seen that $\left(S_{F}, n_{F}\right)$ is topologically equivalent to $(S, n)$.

Thus it is seen that the assertions (i) and (ii) are true in the case $S$ has no boundary components. As for (iii), it follows by corollary 2.4.3 that the topological character of the pointed surface $\left(S_{F}, n_{F}\right)$ determines the group $F$ up to an isomorphism. Thus in cases where $\left(S_{F}, n_{F}\right)$ is of the type (a), (b) or (c) the elements of infinite order (if they exist) form a subgroup that is infinite cyclic, and, hence in these cases $\operatorname{Fix}(F)$ contains at most two points, and cannot contain a free subgroup with two generators. If $\left(S_{F}, n_{F}\right)$ is not of the type (a), (b) or (c) then it is seen that either

(1) $F$ is a free product of more than two cyclic groups, or

(2) $F$ is a free product of two cyclic groups, not both of them isomorphic to $\mathbf{Z}_{2}$.

It follows that $F$ contains a free subgroup with two generators. Thus we have proved theorem 2.8 in case the boundary of $S$ is empty.

It remains to consider the case where the boundary of the pointed surface $(S, n)$ is non-empty. We have defined earlier the double surface $\left(S^{*}, n^{*}\right)$ of $(S, n)$ without boundary. We defined also the surjection $p^{*}: S^{*} \rightarrow S$ and the involution $P: S^{*} \rightarrow S^{*}$. We have seen above that there is a discrete group of the unit disk $F^{*}$ such that $\left(S^{*}, n^{*}\right)$ is topologically equiralent to $\left(S_{F^{*}}, n_{F^{*}}\right)$. We denote the canonical projection $E \rightarrow S^{*}=S_{F^{*}}$ by $p^{\prime}$. Then we have the combined projection

$$
p=p^{*} p^{\prime}: E \rightarrow S .
$$

We have now reached the point where the conclusion may be drawn by corollary 2.4.1. In order to be able to use this corollary, we choose some conformal structure on $S$ (this can be done using a trick employed in Ahlfors-Sario [1] theorem 5E p. 127). This induces a conformal structure on $S^{*}$ such that $p^{*}$ is a local conformal equivalence except at the points of $\partial S$, where it is dihedral of degree one. This induced conformal structure may be different from that of $S^{*}$ regarded as the factor space $S_{F^{*}}$ of $E$. Thus the mapping $p: E \rightarrow(S, n)$ is not, as a rule, a covering projection, 
but it is easy to see that the conformal structure of $E$ may be redefined so that $p$ is a covering projection of the pointed Riemann surface $(S, n)$. Then by corollary 2.4.1 the conformal equivalences $f$ of $E$ for which the triangle

$$
\underset{p_{\searrow}}{E \underset{ }{\rightarrow}} \stackrel{f}{\rightarrow} E
$$

commutes, form a discrete group $F$ of the unit disk such that $\left(S_{F}, n_{F}\right)$ $=(S, n)$. We must still show that the conformal structure of $E$ after the redefinition remains conformally equivalent with the conformal structure of the open unit disk. If $F^{*} \subset F$ contains a free subgroup with two generators, denoted by $H$, then $E / H$ is homeomorphic to either a torus punctured in one point or a sphere punctured in three points (we mar assume that $H$ does not contain indirectly conformal elements). This means that the conformal structure of $E$ is equivalent to that of the open unit disk after the redefinition. In cases where $\left(S^{*}, n^{*}\right)$ is one of the pointed surfaces in (a), (b) or (c), by considering the cases that may occur, we may choose the conformal structure so that the conformal structure of $E$ induced by $p$ is the original conformal structure. Thus we have proved that every non-compact pointed surface may be represented by means of a discrete group of the unit disk.

To conclude the proof of theorem 2.8 we must examine the algebraic structure of $F$. It has a subgroup $F^{*}$ of which we know that it is a free product of cyclic groups. Now, consider the commuting diagram

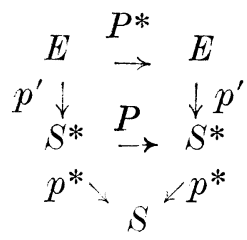

where we choose $P^{*}$ to be a reflection of $F$. It is easy to see, using proposition 2.4, that every element $S \in F$ may be written uniquely in the form $T X$ where $T \in F^{*}$ and $X=P^{*}$ or $=i d$, i.e. is an element of the subgroup generated by $P^{*}$ that is isomorphic to $\mathbf{Z}_{2}$. Thus as a set, the group $F$ can be identified with the cartesian product $F^{*} \times \mathbf{Z}_{2}$, but its composition is not that of the cartesian product. We have

$$
(S X)(T Y)=(S X T X) X Y, S, T \in F^{*} \text { and } X, Y \in \mathbf{Z}_{2}=\left\{P^{*}, i d\right\} .
$$

Thus we may define a left action of $\mathbf{Z}_{2}$ on $F$ by $(X, S) \rightarrow X S X=S^{X}$. Then the group structure of $F$ is given by 


$$
(S, X)(T, Y)=\left(S T^{X}, X Y\right)
$$

We say that this is the skew product structure defined by the action of $\mathbf{Z}_{2}$ on $F^{*}$.

To prove the assertion (iii) it suffices to remark that if $\left(S^{*}, n^{*}\right)$ is topologically equivalent to one of the surfaces in (a), (b) or (c), then every element $T$ of $F$ having infinite order satisfies the condition $T^{2} \in F^{*}$ and is thus in the infinite cyclic subgroup of the elements of $F^{*}$ having infinite order. On the other hand, if $\left(S^{*}, n^{*}\right)$ is not topologically equivalent to one of the surfaces in (a), (b) or (c) then $F^{*}$ and, a fortiori, $F$ contains a free subgroup with two generators. This concludes the proof of the theorem.

We have also proved the following corollary:

2..8.1 Corollary. Let $F$ be a discrete group of a simply connected Riemann surface such that $\left(S_{F}, n_{F}\right)$ is a non-compact pointed surface. If $S_{F}$ has no boundary, $F$ is a free product of cyclic groups. If the boundary of $S_{F}$ is non-empty, there is a well-defined subgroup $F^{*}$ of $F$ such that $\left(S_{F^{*}}, n_{F^{*}}\right)$ $=\left(S_{F}^{*}, n_{F}^{*}\right)$. If $P^{*}$ is a reflection of $F$ and $\mathbf{Z}_{2}$ the subgroup of $F$ generated by $P^{*}$, then $F$ is isomorphic to the skew product $F^{*} \times \mathbf{Z}_{2}$ where the action of $\mathbf{Z}_{2}$ on $F^{*}$ is defined by $(X, S) \rightarrow X S X, S \in F^{*}, X \in \mathbf{Z}_{2}$. This result is also valid if $S_{F}$ is compact.

\section{Isomorphisms between discrete groups of the unit disk}

A. Preliminary definitions and propositions. Let $F$ be some discrete group of conformal mappings of the unit disk. We have already defined

$$
\operatorname{Fix}(F)=\{P(T): T \in F, T \text { has infinite order }\}
$$

which is a subset of $S^{1}$. Similarly we set

$$
\operatorname{Ax}(F)=\{\operatorname{Ax}(T): T \in F, T \text { has infinite order }\}
$$

where it might be observed that we have excluded the axes of reflections of $F$.

3.1 Proposition. Let $S, T \in F$ have infinite order. Then $S$ and $T$ have no common fixed points, unless some power of them is in an infinite cyclic subgroup of $F$.

Proof: The case in which $S$ and $T$ have all their fixed points in common is treated by performing a suitable transformation in such a way that the fixcd points are transformed to 0 and $\infty$ of the Riemann sphere (or to $\infty$ if both $S$ and $T$ are parabolic). Therefore we may assume that $T$ is hyperbolic and has a fixed point that is not a fixed point of $S$. 
Under these circumstances define $S^{\prime}=S T S^{-1}$. Then

$$
d_{T}=d(x, T(x))=d_{S^{\prime}}=d\left(y, S^{\prime}(y)\right), x \in \operatorname{Ax}(T), y \in \operatorname{Ax}\left(S^{\prime}\right)
$$

where $d$ is the hyperbolic metric of $E$, and both $S^{\prime}$ and $T$ are hyperbolic and have one (and only one) common fixed point. Define

$$
T_{n}=T^{n} S^{\prime} T^{-n},
$$

then

$$
d_{T_{n}}=d\left(x, T_{n}(x)\right)=d_{T} \text { for } x \in \operatorname{Ax}\left(T_{n}\right)
$$

Because $\operatorname{Ax}\left(T_{n}\right)$ converges to $\operatorname{Ax}(T)$, we have by (1) and (2)

$$
\lim _{n \rightarrow \infty} T_{n}(z)=T(z) \text { for } z \in \operatorname{Ax}(T) .
$$

But this is impossible since $F$ is discrete and none of the mappings $T_{n}$ is equal to $T$.

Let $f$ be a homeomorphism $\left(S_{F}, n_{F}\right) \rightarrow\left(S_{F^{\prime}}, n_{F^{\prime}}\right)$ where $F$ and $F^{\prime}$ are discrete groups of conformal mappings of simply connected Riemann surfaces $X$ and $X^{\prime}$. In corollary 2.4.3 it was shown that $f$ induces a family of homeomorphisms $X \rightarrow X^{\prime}$ differing from each other by some $S \in F^{\prime}$. At the same time $f$ induces a family of isomorphisms $F \rightarrow F^{\prime}$. The study of such isomorphisms is precisely the subject-matter of this chapter when $X=X^{\prime}=E$. Therefore we define:

3.2 Definition. Let $F$ and $F^{\prime}$ be two discrete groups of conformal mappings of the unit disk and $\varphi: F \rightarrow F^{\prime}$ an isomorphism. We say that $\varphi$ is geometric if there is a homeomorphism $f: E \rightarrow E^{\prime}$ such that

$$
f(T(x))=\varphi(T)(f(x)) \text { for } T \in F, x \in E .
$$

In that case we say that $\varphi$ is induced by $f$.

Our aim is to give necessary and sufficient conditions for an isomorphism of discrete groups of the unit disk to be geometric. In this connexion the following definition is of fundamental importance:

3.3 Definition. Let $F$ be a group of conformal mappings of $E$ and $S, T \in F$ elements of infinite order. Then we say that $S$ and $T$ are crossed if $\operatorname{Ax}(T) \cap \operatorname{Ax}(S) \neq \varnothing$.

It turns out that an isomorphism between discrete groups of $E$ is geometric, with few exceptions, if and only if it preserves the relation of being crossed. We first prove:

3.4 Lemma. Let $F$ and $F^{\prime}$ be two discrete groups of conformal mappings of the unit disk and $\varphi: F \rightarrow F^{\prime}$ a geometric isomorphism. Then $\varphi$ preserves the relation of being crossed.

Proof: It suffices to show that if $\varphi(S)$ and $\varphi(T)$ are crossed for some 
$S, T \in F$, then also $S$ and $T$ are crossed. Moreover, we may assume that neither $\varphi(S)$ nor $\varphi(T)$ is parabolic or that some power of them is in an infinite cyclic subgroup of $F^{\prime}$, i.e. they have a common fixed point. (We suppose that their order is infinite). Let $C_{T}$ be a closed circle arc in $E^{1}$ whose endpoints are $P(T)$ and $N(T)$ (in case $T$ is parabolic let $C_{T}$ be a circle in $E^{1}$, different from $S^{1}$, tangent to $S^{1}$ at $\left.P(T)\right)$. Define similarly $C_{S}$. There is a homeomorphism $f_{T}: \mathbf{R} \rightarrow C_{T} \cap E$ such that

$$
f_{T}(x+n)=T^{n} f(x) \text { for } n \in \mathbf{Z} \text { and } x \in \mathbf{R} .
$$

The homeomorphism $f_{S}: \mathbf{R} \rightarrow C_{S} \cap E$ is constructed in the same way. Let $\overline{\mathbf{R}}=\mathbf{R} \cup\{\infty,-\infty\}$. Then we can extend $f_{T}$ to a continuous map $f_{T}^{\prime}: \overline{\mathbf{R}} \rightarrow C_{T}$ by setting $f_{T}^{\prime}(-\infty)=N(T), f_{T}^{\prime}(\infty)=P(T)$. In the same manner we extend $f_{S}$ to $f_{S}^{\prime}$. Let $h: E \rightarrow E$ be some homeomorphism inducing $\varphi$. Then we define $g_{T}^{\prime}$ and $g_{S}^{\prime}: \overline{\mathbf{R}} \rightarrow E^{1}$ by setting

$$
\begin{aligned}
& g_{T}^{\prime} \mid \mathbf{R}=h f_{S} \\
& g_{T}^{\prime}(-\infty)=N(\varphi(T)), g_{T}^{\prime}(\infty)=P(\varphi(T))
\end{aligned}
$$

and analogously for $g_{S}^{\prime}$. Then from the assumptions we have made regarding $\varphi(T)$ and $\varphi(S)$ it follows that $g_{T}^{\prime}(\overline{\mathbf{R}})$ and $g_{S}^{\prime}(\overline{\mathbf{R}})$ are Jordan ares whose intersection is a point of $E$. Consequently, $C_{T}$ and $C_{S}$ must be circle arcs whose intersection is a point of $E$. This proves that $S$ and $T$ are crossed.

B. The case in which $\mathbf{F i x}(\mathbf{F})$ is dense in $\mathbf{S}^{\mathbf{1}}$. We assume that $F$ and $F^{\prime}$ are two discrete groups for which $\operatorname{Fix}(F)$ and $\operatorname{Fix}\left(F^{\prime}\right)$ are dense in $S^{1}$, and that an isomorphism $\varphi: F \rightarrow F^{\prime}$ preserving the relation of being crossed is given. In that case an element $T$ of $F$ is parabolic if and only if $\varphi(T) \in F^{\prime}$ is parabolic. This is seen using proposition 1.4. Then a mapping $\varphi^{\prime}: \operatorname{Fix}(F) \rightarrow \operatorname{Fix}\left(F^{\prime}\right)$ is defined by

$$
\varphi^{\prime}(P(T))=P(\varphi(T)) .
$$

This is obviously a bijection. The following proposition shows that it can be extended to a homeomorphism $\varphi^{*}: S^{1} \rightarrow S^{1}$, if $\varphi$ preserves the relation of being crossed.

3.5 Proposition. Let $F, F^{\prime}, \varphi$ and $\varphi^{\prime}$ be as above. Then there is a unique extension of $\varphi^{\prime}$ to a homeomorphism $\varphi^{*}: S^{1} \rightarrow S^{1}$.

Proof: In proving the above proposition we make use of the notion of a nested sequence $T_{1}, T_{2}, \ldots$ of elements of $F$. We say that a sequence $T_{1}, T_{2}, \ldots$ of hyperbolic elements of $F$ is a nested sequence if there is a properly decreasing sequence $I_{1} \supset I_{2} \supset \ldots$ of closed intervals of the circle $S^{1}$, such that the endpoints of $I_{i}$ are $P\left(T_{i}\right)$ and $N\left(T_{i}\right), \quad i \geq 1$. 
Using proposition 1.4 it is seen that a sequence $T_{1}, T_{2}, \ldots$ of elements of $F$ is a nested sequence if and only if $\varphi\left(T_{1}\right), \varphi\left(T_{2}\right), \ldots$ is a nested sequence of elements of $F^{\prime}$. Let $x$ be an arbitrary point of $S^{1}$. Then there is a nested sequence $T_{1}, T_{2}, \ldots$ of elements of $F$ such that, if $I_{1}, I_{2}, \ldots$ is the corresponding sequence of intervals,

$$
\{x\}=\bigcap_{i \geq 1} I_{i} .
$$

Then the intersection of intervals corresponding to the nested sequence $\varphi\left(T_{1}\right), \varphi\left(T_{2}\right), \ldots$ consists of one point $y$. We define

$$
\varphi^{*}(x)=y \text {. }
$$

It is easily seen that $\varphi^{*}$ is uniquely determined and is the desired extension of $q^{\prime}$. We may note that $q^{*}$ has the property

$$
q^{*}(T(x))=q(T)\left(q^{*}(x)\right) \text { for } x \in S^{1} \text { and } T \in F .
$$

This is the fundamental property of $\varphi^{*}$ and it will be needed later.

We prove a corollary to proposition 3.5 for which we have no use, but which is, nonetheless, interesting in itself.

3.5.1 Corollary. Let $F, F^{\prime}, \varphi$ and $\varphi^{*}$ be as in proposition 3.5 and $f$ some homeomorphism inducing $\phi$. Then the bijection $E^{1} \rightarrow E^{1}$ defined by $f$ and $\varphi^{*}$ is a homeomorphism.

Proof: The mapping $f^{\prime}: E^{1} \rightarrow E^{1}$ defined by

$$
\begin{aligned}
f^{\prime}(x) & =f(x) \text { for } x \in E \\
& =\phi^{*}(x) \text { for } x \in S^{1}
\end{aligned}
$$

is obviously a bijection and to show that it is a homeomorphism it suffices to prove its continuity at points of $S^{1}$. Let $x$ be a point of $S^{1}$. Let $T^{1}$, $T_{2}, \ldots$ be a nested sequence of elements of $F$ and $I_{1} . I_{2} \ldots$ the sequence of intervals corresponding to it satisfying

$$
\{x\}=\bigcap_{i \geq 1} I_{i} .
$$

Let $U_{i}$ be the component of $E^{1} \backslash \operatorname{Ax}\left(T_{i}\right)$ to which $x$ belongs. Then $U_{i}, i \geq 1$, form a basis of neighbourhoods for $x$ in $E^{1}$. and it is not difficult to see that $f^{\prime}\left(U_{i}\right), i \geq 1$, is a basis of neighbourhoods for $f^{\prime}(x)$.

C. The main theorem. We have now at our disposal all that is needed to prove the main theorem of this chapter. We suppose that two discrete groups $F$ and $F^{\prime}$ of conformal mappings of the unit disk and an isomorphism $\varphi: F \rightarrow F^{\prime}$ are given. We prove, with some restrictions, that $\varphi$ is induced by a homeomorphism if and only if it preserves the relation 
of being crossed. We suppose that the factor space $E / F$ is non-compact, except if the boundary of $S$ is non-empty in which case we assume that boundary components are compact. There are two reasons for this: If $\partial S=\varnothing$, our method of proof applies only when $S$ is non-compact, whereas the case $\partial S \neq \varnothing$ is reduced to this by omitting the boundary. The other reason is that for the case $S$ is compact and $\partial S=\emptyset$ proofs of combinatory nature are available (see e.g. Zieschang-Vogt-Coldewey [7] or Zieschang [6]) but if $\partial S \neq \varnothing$ only proofs making use of quasiconformal theory and Teichmüller spaces are known (cf. Macbeath [3]). The compact case is basically different from the non-compact case in the respece that every isomorphism is induced by a homeomorphism (and thus preserves the relation of being crossed, as we later show in lemma 3.6.2).

3.6 Theorem. Let $F$ and $F^{\prime}$ be two discrete groups of conformal mappings of the unit disk such that $F$ contains a free subgroup with two generators and $S=E / F$ is non-compact or that the boundary of $S$ is non-empty. The boundary components of $S$ are assumed compact. Let $\varphi: F \rightarrow F^{\prime}$ be an isomorphism. Then $\varphi$ is geometric if and only if it preserves the relation of being crossed.

Remark 1. The theorem is presumably true even without the assumption of compactness of the boundary components, but I have not been able to prove it in this case.

Remark 2. If $S$ does not contain a free subgroup with two generators rather strong limitations are imposed on the topological character of the pointed surface $\left(S_{F}, n_{F}\right)$ (cf. theorem 2.8.).

Proof: We agree to call in this proof a discrete group of conformal mappings of the unit disk simply a discrete group. By lemma 3.4 it suffices to show that if $\varphi$ preserves the relation of being crossed it is geometric. We divide the proof in several parts:

\section{Some general results}

We first prove some results of general character for later use.

A. If a discrete group $G$ has a compact fundamental domain. then the set $\operatorname{Fix}(G)$ is dense in $S^{1}$.

In proving this we make use of the following result: If a discrete group $G$ has compact fundamental domain, then it contains a subgroup $G^{\prime}$ of finite index that is a surface group, i.e. $E$ is a covering surface of $E / G^{\prime}$. (This result is proved e.g. in Zieschang-Vogt-Coldewey [7] Satz IV.17). Then $G^{\prime}$ has also a compact fundamental domain and evidently $G^{\prime} \neq\{1\}$. Let $S \neq 1$ be an element of $G^{\prime}$. Then $S$ is a hyperbolic transformation of the unit disk. Let $x$ be an arbitrary point of $S^{1}$ and let $U$ be an open neighbourhood of $x$ in $E^{1}$ whose boundary in $E^{1}$ is a closed are of a circle orthogonal to the unit circle. Then there is an element $T$ of $G^{\prime}$ 
such that $T(\operatorname{Ax}(S)) \cap U \neq \varnothing$, and consequently, $T S T^{-1}$ has a fixed point in $U \cap S^{1}$. This proves $\mathrm{A}$.

$B$. If the isomorphism $\varphi$ preserves the relation of being crossed, then $\varphi$ carries reflections bijectively onto reflections.

To prove $\mathrm{B}$ we choose a reflection $S \in F$. Since $F$ contains a free subgroup with two generators, we deduce using proposition 1.4 (and possibly proposition 3.1) that there is a hyperbolic $T \in F$ such that $\operatorname{Ax}(T) \cap$ $\operatorname{Ax}(S) \neq \varnothing$ or, equivalently, that $T$ and $S T S$ are crossed. Besides we may assume that $S T S \neq T^{-1}$. Then $\varphi(T)$ and $\varphi(S T S)$ are crossed, and since $\varphi(T)^{-1} \neq \varphi(S T S)$ and $\varphi(S)$ is of order two, this is possible only if $\varphi(S)$ is a reflection. Similarly, it can be shown that if $S^{\prime} \in F^{\prime}$ is a reflection then $\varphi^{-1}\left(S^{\prime}\right)$ is a reflection.

\section{The case where $F$ contains no reflections}

If $F$ contains no reflections, then $\mathrm{B}$ shows that $F^{\prime}$ cannot contain reflections, either. Besides, we can suppose using lemma 3.4 and theorem 2.8, that $\operatorname{Fix}(F)$ is dense in $S^{1}$. Similarly, we can assume that $\operatorname{Fix}\left(F^{\prime}\right)$ is dense in $S^{1}$. This is seen as in the case of $F$ if $E / F^{\prime}$ is non-compact or by result $\mathrm{A}$ if it is compact. Besides by the above arguments we can assume that $F$ is a free combination of cyclic groups formed by $F_{i}, D_{i}, T_{i}, i \in K$. In addition notations $P_{i}, N_{i}$ and $C_{i}$ are used as in section $\mathrm{C}$ of chapter 2 . Then according to definition $2.5^{\prime}$ we have the inclusions

$$
\operatorname{cl}\left(C_{i}\right) \cap S^{1} \subset \operatorname{cl}\left(D_{j}\right) \cap S^{1} \text { for } i, j \in K, i \neq j
$$

and the inclusion is proper if $F_{i}$ and $F_{j}$ are cyclic groups of order two.

Since both $\operatorname{Fix}(F)$ and $\operatorname{Fix}\left(F^{\prime}\right)$ are dense in $S^{1}$ and $q$ preserves the relation of being crossed there is a homeomorphism $q^{*}: S^{1} \rightarrow S^{1}$ satisfying (2)

$$
\begin{aligned}
& \varphi^{*}(P(T))=P(\varphi(T)) \text { for } T \in F \text { the order of } T \text { infinite, } \\
& \varphi^{*}(T(x))=\varphi(T)\left(\varphi^{*}(x)\right) \text { for } x \in S^{1} \text { and } T \in F
\end{aligned}
$$

by proposition 3.5. We use $\varphi^{*}$ to construct $F^{\prime}$ as a free combination of cyclic groups.

Let $i$ be an element of $K$ such that $F_{i}$ is infinite. Then the fundamental domain $D_{i}$ of $F_{i}$ is a closed region of $E$ whose boundary consists of two non-Euclidean lines $P_{i}$ and $N_{i}$. $F_{i}$ has a generator $T_{i}$ such that $T_{i}\left(N_{i}\right)=P_{i}$ and $P\left(T_{i}\right)$ and $N\left(T_{i}\right) \in \mathrm{cl} C_{i}$. The non-Euclidean line $P_{i}$ has two (ideal) endpoints $x$ and $y$. Then there is a well-defined non-Euclidean line whose (ideal) endpoints are $\psi^{*}(x)$ and $\varphi^{*}(y)$. It is denoted by $P_{i}^{\prime}$ and the non-Euclidean line $N_{i}^{\prime}$ is defined similarly. If $T_{i}^{\prime}=\varphi\left(T_{i}\right), \quad T_{i}^{\prime}\left(N_{i}^{\prime}\right)=P_{i}^{\prime}$ and $P\left(T_{i}^{\prime}\right), N\left(T_{i}^{\prime}\right) \in \psi^{*}\left(\mathrm{cl} C_{i} \cap S^{1}\right)$. This means 
that the closed region of $E$, denoted by $D_{i}^{\prime}$, whose boundary consists of the lines $P_{i}^{\prime}$ and $N_{i}^{\prime}$ is a fundamental domain for $F_{i}^{\prime}=\varphi\left(F_{i}\right)$.

If the order of $F_{i}$ is finite we proceed quite similarly. It is a consequence of the equations (2) that if $T_{i}$ is a generator of $F_{i}$ that is a rotation through the angle $2 \pi / n$ then $T_{i}^{\prime}=\varphi\left(T_{i}\right)$ is also a rotation through the angle $2 \pi / n$. Then $T_{i}$ satisfies the condition $P_{i}=T_{i}\left(N_{i}\right)$ where $P_{i}$ and $N_{i}$ are the sides of the fundamental domain $D_{i}$ of $F_{i}$. We associate with the non-Euclidean ray $P_{i}$ another non-Euclidean ray $P_{i}^{\prime}$ as follows: $P_{i}$ has an (ideal) endpoint $x$ in $S^{1}$. The other endpoint of $P_{i}$ is the fixed point of $T_{i}$. Let $P_{i}^{\prime}$ be the non-Euclidean ray whose (ideal) endpoint is $\varphi^{*}(x)$ and the other endpoint the fixed point of $T_{i}^{\prime}$. We define similarly $N_{i}^{\prime}$. Let $D_{i}^{\prime}$ be the closed region of $E$ whose boundary is $P_{i}^{\prime} \cup N_{i}^{\prime}$ and such that $\varphi^{*}\left(\operatorname{cl} C_{j} \cap S^{1}\right) \subset \operatorname{cl} D_{i}^{\prime}, j \neq i, j \in K$. Since the index set $K$ contains at least two elements, $D_{i}^{\prime}$ is defined uniquely by this requirement. It is clear that $D_{i}^{\prime}$ is a fundamental domain for $F_{i}^{\prime}=$ $p\left(F_{i}\right)$.

We claim that $F^{\prime}$ is formed by the system $F_{i}^{\prime}, D_{i}^{\prime}, T_{i}^{\prime}, K$ as a free combination of cyclic groups. Let $C_{i}^{\prime}=E \backslash D_{i}^{\prime}$ for $i \in K$. Applying $\varphi^{*}$ to the inclusions (1) we obtain

$$
\operatorname{cl} C_{i}^{\prime} \cap S^{1} \subset \operatorname{cl} D_{j}^{\prime} \cap S^{1} \text { for } i, j \in K, i \neq j
$$

and the inclusion is proper if $F_{i}^{\prime}$ and $F_{j}^{\prime}$ are cyclic groups of order two. Then according to the definition $2.5^{\prime}, F_{i}^{\prime}, D_{i}^{\prime}, T_{i}^{\prime}, K$ forms a free combination of cyclic groups. Since $\operatorname{Fix}\left(F^{\prime}\right)$ is dense in $S^{1}, D^{\prime}=\bigcap_{i \in K} D_{i}^{\prime}$ is a funda-
mental domain for $F_{j}^{\prime}$ by proposition 2.6 .

Obviously there is a homeomorphism $h$ from the fundamental domain $D=\bigcap_{i \in K^{\prime}} D_{i}$ of $F$ to the fundamental domain $D^{\prime}=\bigcap_{i \in K} D_{i}^{\prime}$ of $F^{\prime}$ such that $h\left(T_{i}(x)\right)=T_{i}^{\prime}(h(x))$ for $i \in K$ and $x \in N_{i}$. Then we can define a homeomoxphism $f: E \rightarrow E$ inducing $\varphi$ by setting $f(T(x))=\varphi(T)(h(x))$ for $x \in D$ and $T \in F$. Clearly, this is the desired homeomorphism.

\section{The case with non-empty boundary}

We now proceed to the case in which the boundary is non-empty while still assuming that boundary components are compact. This means that there are reflections in the groups $F$ and $F^{\prime}$. We generalize the definition of being crossed also to the case in which $S$ and $T$ are reflections: We say that two distinct reflections are crossed if their axes intersect. The principal difference between the above definition and the definition of being crossed for elements of infinite order is that a reflection is not crossed with itself. The following assertion is trivial: 
C. Two distinct reflections in a discrete group are crossed if and only if their product is of finite order.

Let $R, S$ and $T$ be reflections. We say that $S$ separates $R$ and $T$ if $\operatorname{Ax}(R)$ and $\operatorname{Ax}(T)$ lie in different components of $E^{1} \backslash \mathrm{Ax}(S)$.

$D$. Let $R, S$ and $T$ be reflections of $F$. Then $R^{\prime}=\varphi(R), T^{\prime}=\varphi(T)$ are separeted by $S^{\prime}=\varphi(S)$ if and only if $S$ separates $R$ and $T$.

We may assume that the axes of $R, S$ and $T$ do not intersect. Then D follows immediately if we observe that $R$ and $T$ are not separated by $S$ if and only if there is a hyperbolic transformation $P$ in $F$ such that the axis of $P$ intersects the axes of $R$ and $T$ but not that of $S$. This is seen using proposition 1.4 and observing that the components of $S^{1} \backslash \operatorname{Ax}(R)$ and $S^{1} \backslash \operatorname{Ax}(T)$ that do not intersect with $\operatorname{Ax}(S)$ contain points of $\operatorname{Fix}(F)$. Similarly, if $S^{\prime}$ separates $R^{\prime}$ and $T^{\prime}, S$ separates $R$ and $T$.

Next we choose a component $E_{0}$ of $p^{-1}(S \backslash \partial S)$ where $p: E \rightarrow S$ is the canonical projection. The boundary of $E_{0}$ in $E$ consists of axes of reflections of $F$, with the endpoints excluded, or closed segments of axes of reflections of $F$.

We denote by $r\left(E_{0}\right)$ the set of reflections in $F$ whose axes intersect the boundary of $E_{0}$; thus

$$
r\left(E_{0}\right)=\left\{S \in F: S \text { is a reflection and } \operatorname{Ax}(S) \cap \mathrm{cl}_{E} E_{0} \neq \varnothing\right\} .
$$

The set $r\left(E_{0}\right)$ satisfies the condition:

$E$. Each element $T$ of $r\left(E_{0}\right)$ is either crossed with no other reflection of $F$ or it is crossed with exactly two reflections $R$ and $S$ in $r(E)$. In the former case $\operatorname{Ax}(T) \cap \mathrm{cl}_{E} E_{0}$ is $\operatorname{Ax}(T) \cap E$; in the latter it is the closed segment of $\operatorname{Ax}(T)$ whose endpoints are the points where $\operatorname{Ax}(T)$ intersects the axes of $R$ and $S$. Besides these endpoints this segment does not contain other fixed points of rotations.

Let $T$ be an element of $r\left(E_{0}\right)$. Then $E_{0}$ is contained in one component of $E \backslash \operatorname{Ax}(T)$. Therefore $\operatorname{Ax}(T) \cap \mathrm{cl} E_{0}$ is a disjoint union of closed segments of $\operatorname{Ax}(T) \cap E$ or is $\operatorname{Ax}(T) \cap E$. It is $\operatorname{Ax}(T) \cap E$ if $p(\operatorname{Ax}(T) \cap E)$ is contained in a boundary component of the pointed surface $(S, n)=$ $\left(S_{F}, n_{F}\right)$ that does not contain points of $n$; this is equivalent to the statement that $T$ is not crossed with other reflections of $F$. If $T$ is crossed with other elements of $F$ then each component of $\operatorname{Ax}(T) \cap \mathrm{cl}_{E} E_{0}$ is a closed segment of $\operatorname{Ax}(T)$ whose endpoints are fixed points of rotations of $F$. Let $A_{T}$ be a component of $\operatorname{Ax}(T) \cap \mathrm{cl}_{E} E_{0}$, and let $A_{T}^{\prime}$ be a subsegment of $A_{T}$ that does not contain fixed points of rotations of $F$ other than its endpoints $x_{1}$ and $x_{2}$. Then there are elements $S_{1}$ and $S_{2}$ of $r\left(E_{0}\right)$ such that $\operatorname{Ax}\left(S_{i}\right) \cap A_{T}^{\prime}=\left\{x_{i}\right\}, i=1,2$. Then $\operatorname{Ax}(T) \backslash\left(C_{1} \cup C_{2}\right)=$ $A_{T}^{\prime} \backslash\left\{x_{1}, x_{2}\right\}$ when $C_{i}$ is the component of $E \backslash \operatorname{Ax}\left(S_{i}\right)$ for which $E_{0} \cap C_{i}$ $=\varnothing, \quad i=1,2$. 
Let $T$ be a reflection of $r\left(E_{0}\right)$. Then we set

$$
A_{T}=\operatorname{Ax}(T) \cap \mathrm{cl}_{E} E_{0} \text {. }
$$

This is by $D$ either the non-Euclidean line $\operatorname{Ax}(T) \cap E$ or a closed segment of $\operatorname{Ax}(T)$ whose endpoints are the fixed points of rotations $R T$ and $S T$ where $R$ and $S$ are the two elements of $r\left(E_{0}\right)$ with which $T$ is crossed. Analogously we define for $T^{\prime}=\varphi(T) \in \varphi\left(r\left(E_{0}\right)\right)$

$$
\begin{aligned}
A_{T^{\prime}}^{\prime}= & \operatorname{Ax}\left(T^{\prime}\right) \cap E \text { if } A_{T}=\operatorname{Ax}(T) \cap E \\
= & \text { the closed segment of } \operatorname{Ax}\left(T^{\prime}\right) \text { whose endpoints are the fixed points } \\
& \text { of rotations } R^{\prime} T^{\prime} \text { and } S^{\prime} T^{\prime} \text { when } R=\varphi^{-1}\left(R^{\prime}\right) \text { and } S=\varphi^{-1}\left(S^{\prime}\right) \\
& \text { are the reflections of } r\left(E_{0}\right) \text { crossed with } T .
\end{aligned}
$$

Then we claim:

$F$. The set $E_{0}$ is the unique open convex subset of $E$ whose boundary in $E$ is

$$
A=\cup A_{T}, T \in r\left(E_{0}\right) \text {. }
$$

Similarly there is a unique open convex subset $E_{0}^{\prime}$ of $E$ whose boundary is

$$
A^{\prime}=\cup A_{T^{\prime}}^{\prime}, T^{\prime} \in \varphi\left(r\left(E_{0}\right)\right) \text {. }
$$

The subgroup $F_{0}$ of $F$ that leaves $E_{0}$ invariant consists of those elements $S$ of $F$ for which the mapping $S \mapsto S T S^{-1}$ is a bijection of $r\left(E_{0}\right)$ onto $r\left(E_{0}\right)$.

Let $F_{0}^{\prime}=\varphi\left(F_{0}\right)$. Then $F_{0}^{\prime}$ is the subgroup $F^{\prime}$ that leaves $E_{0}^{\prime}$ invariant. As above its elements are those elements $S^{\prime}$ of $F^{\prime}$ for which the mapping $T^{\prime} \mapsto S^{\prime} T^{\prime} S^{\prime-1}$ is a bijection of $\varphi\left(r\left(E_{0}\right)\right)$ onto $\varphi\left(r\left(E_{0}\right)\right)$.

To show that $E_{0}$ is the unique open convex domain whose boundary is $A$ we observe that if $r\left(E_{0}\right)$ does not consist of three reflections crossed with each other then for each $T \in r\left(E_{0}\right)$ there is an element $S \in r\left(E_{0}\right)$ such that $S$ and $T$ are not crossed. If $r\left(E_{0}\right)$ contains at least four elements this is seen by $E$. If $r\left(E_{0}\right)$ consists of three or two reflections not crossed with each other, then, evidently, this condition is satisfied. The case that $r\left(E_{0}\right)$ consists of only one element or two elements crossed with each other is impossible, since $F$ contains a free subgroup with two generators. Finally, if $r\left(E_{0}\right)$ consists of three reflections crossed with each other, then $E_{0}$ is the set of interior points of the hyperbolic triangle bounded by the axes of these reflections.

Next we prove that $F_{0}$ has the properties as asserted in $F$. Clearly, each element $T$ of $F_{0}$ leaves the boundary of $E_{0}$ fixed; hence the mapping $S \mapsto T S T^{-1}, S \in r\left(E_{0}\right)$, is a bijection of $r\left(E_{0}\right)$ onto itself. If $T$ is an element of $F$ and the mapping $S \mapsto T S T^{-1}, S \in r\left(E_{0}\right)$, is a bijection of $r\left(E_{0}\right)$ onto itself, then $T$ leaves the boundary of $E_{0}$ fixed and by the argument of the preceding paragraph $T$ leaves $E_{0}$ invariant. 
It remains to prove the assertions concerning $E_{0}^{\prime}$ and $F_{0}^{\prime}$. If there is an open convex set $E_{0}^{\prime}$ whose boundary is $A^{\prime}$, then $E_{0}^{\prime}$ must be unique. This is seen as in the case of $E_{0}$. To prove that there is an open convex set whose boundary is $A^{\prime}$ it suffices by $E$ to show that there are no elements $R^{\prime}, S^{\prime}$ and $T^{\prime}$ of $\varphi\left(r\left(E_{0}\right)\right)$ such that $S^{\prime}$ separates $R^{\prime}$ and $T^{\prime}$. Then by $D$, if $R=\varphi^{-1}\left(R^{\prime}\right), S=\varphi^{-1}\left(S^{\prime}\right)$ and $T=\varphi^{-1}\left(T^{\prime}\right), S$ would separate $R$ and $T$ which is impossible as $S, R, T \in r\left(E_{0}\right)$.

The assertions concerning $F_{0}^{\prime}$ are proved like the assertions concerning $F_{0}$.

Since $E_{0}$ is convex by assertion $F$, it is simply connected. Then we have by corollary 2.4.1:

G. If $F_{0}$ is regarded as a group of conformal mappings of the simply connected Riemann surface $E_{0}$, then $\left(S_{F_{0}}, n_{F_{0}}\right)$ may be identified with the pointed surface $(S \backslash \partial S, n \mid S \backslash \partial S)$.

In particular, since $S \backslash \partial S$ does not have boundary, an element of $F_{0}$ regarded as a mapping of $E_{0}$ cannot be a reflection. We can draw from this the conclusion that an element of $F_{0}$ regarded as a mapping of $E$ is not a reflection.

We denote the closure of $E_{0}$ in $E$ by $\bar{E}_{0}$ and that of $E_{0}^{\prime}$ by $\bar{E}_{0}^{\prime}$. We claim:

H. There is a homeomorphism $f: \bar{E}_{0} \rightarrow \bar{E}_{0}^{\prime}$ such that

$$
f(T(x))=\varphi(T)(f(x)) \text { for } x \in \bar{E}_{0} \text { and } T \in F_{0}
$$

and such that $f \mid A_{T}$ is a homeomorphism $A_{T} \rightarrow A_{r(T)}^{\prime}$ for $T \in r\left(E_{0}\right)$ where $A_{T}$ and $A_{\varphi(T)}^{\prime}$ are as in $F$.

We consider first the types of boundary components in $E$ that $E_{0}$ may have. First of all, $\bar{E}_{0}$ may be compact. Then $\bar{E}_{0}$ is a hyperbolic polygon whose boundary is a finite union $\bigcup_{i=1}^{n} A_{T_{i}}$ where $T_{1}, \ldots, T_{n}, T_{1}$ are the reflections of $r\left(E_{0}\right)$ such that each reflection is crossed with the preceding one and $n$ is an integer $\geq 3$. Then we may say that the boundary of $\bar{E}_{0}$ (and the corresponding boundary component) is closed.

If $\bar{E}_{0}$ is not compact two types of boundary components may occur. Each component of the boundary of $E_{0}$ in $E$ is either of the form $A_{T}=$ $\operatorname{Ax}(T) \cap E$ for a reflection $T$ of $r\left(E_{0}\right)$ or it is a chain of segments of the form $\bigcup_{i=-\infty}^{\infty} A_{T_{i}} \quad$ where in the sequence ..., $T_{-1}, T_{0}, T_{1}, \ldots$ of elements of $r\left(E_{0}\right)$ each reflection is crossed with the preceding one and $T_{i} \neq T_{j}$ for $i \neq j$. In the former case, $\operatorname{Ax}(T)$ is also the axis of a hyperbolic transformation of $F$ by the compactness of the boundary component $p(\operatorname{Ax}(T) \cap E)$ of $S$. It is called a simple axis. By the same argument, in the latter case there is a hyperbolic transformation $T$ of $F$ such that $T_{p+n}=T T_{n} T^{-1}$ 
for some $p \in \mathbf{Z}$ and all $n \in \mathbf{Z}$. In this case the boundary component is called a chain of segments.

We first consider the following special cases which correspond to cases (a), (b) and (c) of (ii) in theorem 2.8, as we shall see.

(a) $F_{0}$ is either finite cyclic or trivial.

(b) $F_{0}$ is infinite cyclic.

(c) $F_{0}$ is a free product of two cyclic groups of order two.

(a) $F_{0}$ is finite cyclic or trivial. In this case the discussion in the first two paragraphs following assertion $H$ shows that then $\bar{E}_{0}$ must be compact. The set $r\left(E_{0}\right)$ consists of $n$ reflections $T_{i}, 1 \leq i \leq n$, where $n \geq 3$ and in the sequence $T_{1}, \ldots, T_{n}, T_{1}$ each reflection is crossed with the preceding one. Let $T$ be a generator of the group $F_{0}$ that is a rotation through the angle $2 \pi / k$ where $k$ is the order of the group $F_{0}$. T leaves the boundary of $E_{0}$ fixed and hence $E_{0}$ is also fixed under $T$. It follows (using e.g. the Brouwer fixed point theorem) that $T$ has a fixed point in $E_{0}$.

Let $T_{i}^{\prime}=\varphi\left(T_{i}\right), 1 \leq i \leq n$. Then each element in the sequence $T_{1}^{\prime}, \ldots, T_{n}^{\prime}, T_{1}^{\prime}$ is crossed with the preceding one. The segments $A_{T_{i}^{\prime}}^{\prime}$, $1 \leq i \leq n$, form the boundary of the polygon $\bar{E}_{0}^{\prime}$. Since $T^{\prime}=\varphi(T)$ leaves the boundary of $\bar{E}_{0}^{\prime}$ invariant, it is seen as above that $T^{\prime}$ must be a rotation that has a fixed point in $E_{0}^{\prime}$. In addition $T^{\prime \prime}$ is a rotation through the angle $2 \pi / k$. This follows from the fact that $k$ divides $n$ and if $m=n / k$, then $T_{m+1}=T T_{1} T^{-1}$, and so $T_{m+1}^{\prime}=T^{\prime} T_{1}^{\prime} T^{\prime-1}$ (if $m=n$, we set $T_{m+1}=T_{1}$ and $\left.T_{m+1}^{\prime}=T_{1}^{\prime}\right)$.

Now that we have clarified the situation it is easy to see that there is such a homeomorphism as asserted in $H$.

(b) $F_{0}$ is infinite cyclic. We may regard $F_{0}$ as a group of conformal mappings of $E_{0}$. Then we may identify the pointed surfaces $\left(S_{F_{0}}, n_{F_{0}}\right)$ and $(S \backslash \partial S, n \mid S \backslash \partial S)$. From theorem 2.8 we have that $n_{F_{0}} \equiv 1$ and $S \backslash \partial S$ is topologically equivalent to either a sphere punctured at two points or to a projective plane punctured at one point. Let $T$ be the generator of $F_{0}$. In the former case $S$ is either non-compact and has one boundary component (and one point of ideal boundary) or it is compact and has two boundary components. If $S$ is non-compact it is easy to see that $E_{0}$ has one boundary component, which must be, since we assume that $F$ contains a free subgroup with two generators, a chain of segments. This chain of segments has as accumulation points the fixed points $P(T)$ and $N(T)$ of $T$. If $S$ is compact it is seen as above that $E_{0}$ has two boundary components of which at least one is a chain of segments. Both boundary components have $P(T)$ and $N(T)$ as their accumulation points and both are invariant under $F_{0}$. If $S \backslash \partial S$ is a projective plane punctured at one point, then $S$ is compact and has one boundary component. 
It is easy to see that in this case $E_{0}$ has two boundary components that are chains of segments having $P(T)$ and $N(T)$ as their accumulation points and such that $T$ interchanges them.

Since the structure of $r\left(E_{0}\right)$ with respect to the relation of being crossed and the action of $F_{0}$ defined by $S \mapsto T S T^{-1}, S \in r\left(E_{0}\right), T \in F_{0}$ is preserved under the bijections $r\left(E_{0}\right) \rightarrow \varphi\left(r\left(E_{0}\right)\right)$ and $F_{0} \rightarrow F_{0}^{\prime}$ induced by $q$, it is easily verified that the desired homeomorphism $f$ exists.

(c) $F_{0}$ is the free product of two cyclic groups of order two. We refer the reader to the treatment of case (ii)c in theorem 2.8. Since $F_{0}$ does not contain reflections, $F_{0}$ has as generators two rotations $S$ and $T$ of order two. The transformation $R=S T$ is a hyperbolic transformation whose axis passes through the fixed points of $S$ and $T$. Every element of $F_{0}$ is either a rotation of order two or is in the subgroup generated by $R$. Let the fixed point of $T$ be $P$ and that of $R T R^{-1}$ be $Q=R(P)$. Let $A$ be the closed arc of the axis of $R$ whose endpoints are $P$ and $Q$ and let $B$ and $C$ be two non-Euclidean rays whose endpoints are $P$ and $Q$, orthogonal to the axis of $R$, such that $R(B)=C$. Then one of the two closed regions of $E$ whose boundary is $A \cup B \cup C$ is a fundamental domain for $F_{0}$, regarded as a discrete group of $E$. We denote it by $D$. If we consider $F_{0}$ as a discrete group of $E_{0}$ we may observe that $\operatorname{Ax}(R) \cap E \subset E_{0}$ and $E_{0}$ has two boundary components $C_{1}$ and $C_{2}$ having $P(R)$ and $N(R)$ as accumulation points in $E^{1}$ and such that $R$ carries $C_{i}$ to $C_{i}, i=1,2$, and each rotation of $F_{0}$ interchanges $C_{1}$ and $C_{2}$. Both $C_{1}$ and $C_{2}$ are chains of segments. A fundamental domain of $F_{0}$ as a discrete group of $E_{0}$ is $E_{0} \cap D$. All this follows from what has been said above and from the fact that the pointed surface $(S, n)$ has one boundary component with $n(x)=1$ for all interior points $x \in S$ excepting two interior points $y$ and $z$ for which $n(y)=n(z)=2$. Now the existence of the desired homeomorphism $f$ is easily verified.

After these special cases we consider the general case. The group $F_{\mathbf{0}}$ may be regarded as a discrete group of $E_{0}$ and then $(S \backslash \partial S, n \mid S \backslash \partial S$ ) may be identified with $\left(S_{F_{0}}, n_{F_{0}}\right)$. We wish to show that if $F_{0}$ is not one of the special groups (a), (b) or (c) considered above, then $F_{0}$ contains a free subgroup with two generators. But this will follow immediately from theorem 2.8 if we remember that the above special cases correspond to the cases (a), (b) and (c) of theorem 2.8 and in all other cases a discrete group not containing reflections contains a free subgroup with two generators. But $F_{0}$ does not contain reflections and so we may draw the desired conclusion. Our aim is to show that part 2 of this proof can be used to prove the existence of a homeomorphism $f^{\prime}: E_{0} \rightarrow E_{0}^{\prime}$ satisfying the condition (3) of assertion $H$. We regard $F_{0}$ as a discrete group of $E_{0}$ and $F_{0}^{\prime}$ as a discrete group of $E_{0}^{\prime}$. We could now use part 2 of this proof if $E_{0}$ 
and $E_{0}^{\prime}$ were the unit disk. But the important thing is that they are conformally equivalent with the unit disk. We choose a conformal equivalence $h: E_{0} \rightarrow E$ and transform the group $F_{0}$ using $h$ to the discrete group $F_{1}=\left\{h T h^{-1}: T \in F_{0}\right\}$ of the unit disk. The mapping $T \mapsto h T h^{-1}$ is an isomorphism $\varphi_{1}: F_{0} \rightarrow F_{1}$. We define similarly the discrete group $F_{1}^{\prime}$ of the unit disk and isomorphism $\varphi_{1}^{\prime}: F_{0}^{\prime} \mapsto F_{1}^{\prime}$. Let $\varphi_{0}=\varphi \mid F$. Then $\varphi_{1}^{\prime} \varphi_{0} \varphi_{1}^{-1}: F_{1} \rightarrow F_{1}^{\prime}$ is an isomorphism. If we can show that it preserves the relation of being crossed, the desired homeomorphism $f^{\prime}: E_{0} \rightarrow E_{0}^{\prime}$ exists by part 2 of this proof.

To prove that $\varphi_{1}^{\prime} \varphi_{0} \varphi_{1}^{-1}$ preserves the relation of being crossed it suffices to show that $\varphi_{1}$ and $\varphi_{1}^{\prime}$ preserve this relation when $F_{0}$ and $F_{0}^{\prime}$ are regarded as discrete groups of the unit disk. The proof resembles closely that of lemma 3.4 and is therefore omitted.

We have now shown that there exists a homeomorphism $f^{\prime}: E_{0} \rightarrow E_{0}^{\prime}$ satisfying equation (3) of $H$. By means of it we construct a homeomorphism $f: \bar{E}_{0} \rightarrow \bar{E}_{0}^{\prime}$ where $f$ has the properties as asserted in $H$. The set $\bar{E}_{0} / F_{0}$ may be identified with $S$. Let the boundary components of $S$ be $C_{i}, i \in J$. Then there is a family of disjoint sets $K_{i} \subset S$ and homeomorphisms $f_{i}: S^{1} \times I \rightarrow K_{i}$ such that $f_{i}\left(S^{1} \times\{0\}\right)=C_{i}$ for $i \in J$ (we denote by $I$ the interval $[0,1])$. Moreover, we can suppose that if $x \in S^{\mathbf{1}}$, $i \in J$, and $\left.K_{i}^{\prime}=f_{i}\left(S^{1} \backslash\{x\}\right) \times I\right)$, and $p_{0}: \bar{E}_{0} \rightarrow S$ is the canonical surjection, then $p_{0}$, restricted to a component $U$ of $p_{0}^{-1}\left(K_{i}^{\prime}\right)$, is a homeomorphism $U \rightarrow K_{i}^{\prime}$. To each boundary component $C$ of $\bar{E}_{0}$ there corresponds a unique component $K_{C}$ of $p_{0}^{-1}\left(\bigcup_{i \in J} K_{i}\right)$ such that $K_{C} \cap C=C$. Let $F_{C}$ be the infinite cyclic subgroup of $F_{0}$ carrying $C$ to itself and let $T_{C}$ be a generator of $F_{C}$. Then there is a homeomorphism $f_{C}: \mathbf{R} \times I \rightarrow$ $K_{C}$ such that $f_{C}(x+n, t)=T_{C}^{n}(f(x, t))$ for $n \in \mathbf{Z}, x \in \mathbf{R}$ and $t \in I$. It is clear that in $K_{C}$ only the points $T_{C}^{n}(x), n \in \mathbf{Z}$, are equivalent to $x \in K_{C}$ under the group $F_{0}$. We assume that $C=f_{C}(\mathbf{R} \times\{0\})$.

Each boundary component $C$ of $\bar{E}_{0}$ is a union of segments $A_{T}$ and the union of the corresponding segments $A_{f(T)}^{\prime}$ is a boundary component $C^{\prime}$ of $\bar{E}_{0}^{\prime}$. Then the infinite cyclic subgroup $F_{C^{\prime}}^{\prime}$ of $F_{0}^{\prime}$ carrying $C^{\prime}$ to $C^{\prime}$ is generated by $T_{C^{\prime}}^{\prime}=q\left(T_{C}\right)$. Furthermore, it is clear that the boundary of $f^{\prime}\left(f_{C}(\mathbf{R} \times(0,1])\right.$ has two components in $\bar{E}_{0}^{\prime}$ of which one must be $C^{\prime}$ since otherwise $f^{\prime}\left(f_{C}(\mathbf{R} \times(0,1))\right)$ would be that component of $E_{0}^{\prime} \backslash f^{\prime}\left(f_{C}(\mathbf{R} \times\{1\})\right)$ that has infinite number of boundary components and in $f^{\prime}\left(f_{C}(\mathbf{R} \times(0,1))\right)$ only points of $T_{C^{\prime}}^{\prime n}(x), n \in \mathbf{Z}$, are equivalent with $x$ under $F_{0}^{\prime}$. After that it is seen that there is a homeomorphism $f_{C}^{\prime}: K_{C} \rightarrow K_{C^{\prime}}^{\prime}=\operatorname{cl}_{E} f^{\prime}\left(K_{C} \backslash C\right)$ such that the restrictions of $f_{C}^{\prime}$ and $f^{\prime}$ to $f_{C}(\mathbf{R} \times\{1\})$ are equal and that $f_{C}^{\prime}: A_{T}$ is a homeomorphism $A_{T} \rightarrow A_{\varphi(T}^{\prime}$ for each $A_{T} \subset C$ and that

$$
f_{C}^{\prime}\left(T_{C}^{n}(x)\right)=T_{C^{\prime}}^{\prime n}\left(f_{C}^{\prime}(x)\right) \text { for } n \in \mathbf{Z} \text { and } x \in K_{C} .
$$


Let $B=T(C)$ be another boundary component of $E_{0}$ equivalent to $C$ under $F_{0}$. Then we define

$$
f_{B}^{\prime}(x)=\varphi(T)\left(f_{C}^{\prime}\left(T^{-1}(x)\right)\right) \text { for } x \in K_{B} .
$$

Since the $f_{B}^{\prime}$ and $f^{\prime}$ coincide on $f_{B}(\mathbf{R} \times\{1\})$ we see that $f^{\prime}$ can be modified in the sets $K_{B}, B=T(C), T \in F_{0}$, in such a way that we obtain a homeomorphism $f_{C}^{\prime \prime}: E_{0} \cup\left(\bigcup_{T \in F_{0}} T(C)\right) \rightarrow E_{0}^{\prime} \cup\left(\bigcup_{T^{\prime} \in F_{0}^{\prime}} T^{\prime}\left(C^{\prime}\right)\right)$ satisfying the equation (3) of $H$ and the condition that $f_{C}^{\prime \prime} \mid A_{T}$ is a homeomorphism $A_{T} \rightarrow A_{\varphi(T)}^{\prime}$ whenever $f_{C}^{\prime \prime}$ is defined on $A_{T}$.

It is clear that we can by the above procedure obtain a homeomorphism $f$ satisfying the conditions of assertion $H$.

We proceed by defining inductively a sequence of sets $F_{n} \subset F, n \geq 1$. Let

$$
F_{1}=\left\{S T: S \in r\left(E_{0}\right), T \in F_{0}\right\}
$$

and further, if $F_{n-1}$ has already been defined,

(4) $F_{n}=\left\{S T: T \in F_{n-1}, S=S^{\prime} T^{\prime} S^{\prime-1}\right.$ where $S^{\prime \prime} \in F_{n-1}$ and $\left.T^{\prime} \in r\left(E_{0}\right)\right\}$.

It is clear that

$$
F=\bigcup_{n=1}^{\infty} F_{n} .
$$

Since each $F_{n}, n \geq 1$, is in the subgroup of $F$ generated by $F_{0}$ and $r\left(E_{0}\right)$ we see that $F_{0}$ and $r\left(E_{0}\right)$ generate $F$. Since $\varphi: F \rightarrow F^{\prime}$ is an isomorphism we obtain the result that $F_{0}^{\prime}=q\left(F_{0}\right)$ and $\varphi\left(r\left(E_{0}\right)\right)$ generate $F^{\prime}$.

We are going to show that we can identify the set $\bar{E}_{0}^{\prime} / F_{0}^{\prime}$ with $S^{\prime}=$ $E / F^{\prime}$. In proving this we need:

I. If $T^{\prime}$ is a reflection of $F^{\prime}$, then $E_{0}^{\prime} \cap \mathrm{Ax}(T)=0$.

We first suppose that $r\left(E_{0}\right)$ does not consist of three reflections crossed with each other, i.e. $\bar{E}_{0}$ is not a hyperbolic triangle. We remark that to show that $\bar{E}_{\mathbf{0}}$ is a hyperbolic triangle it suffices to find three reflections $R, S, T$ of $F$ whose axes bound a hyperbolic triangle 1 . For then either $\Delta$ is a component of $p^{-1}(S \backslash \partial S)$ or there is a reflection $R^{\prime}$ of $F$ such that $\operatorname{Ax}\left(R^{\prime}\right) \cap \operatorname{int} A \neq \varnothing$. But then the axes of $R^{\prime}$ and, say $S$ and $T$, would bound a hyperbolic triangle $\Delta^{\prime}$ that is a proper subset of $\Delta$. After a finite number of steps we would have a hyperbolic triangle $\Delta^{\prime \prime}$ bounded by the axes of reflections $R^{\prime \prime}, S^{\prime \prime}, T^{\prime \prime}$ of $F$ that is a component of $p^{-1}(S \backslash \partial S)$ and is a subset of $\Delta$.

We assume that $T^{\prime}$ is a reflection of $F^{\prime}$ for which $\operatorname{Ax}\left(T^{\prime}\right) \cap E \neq \varnothing$. We suppose besides that the axis of $T^{\prime}$ intersects a boundary component of $E_{0}^{\prime}$, which must be a chain of segments. Then there are two possibil- 
ities: First there would be two reflections $R^{\prime}$ and $S^{\prime}$ of $\varphi\left(r\left(E_{0}\right)\right)$ separated by $T^{\prime}$. But this is impossible by $D$ since then there would be two reflections $S$ and $R$ of $r\left(E_{0}\right)$ separated by $\varphi^{-1}\left(T^{\prime}\right)$. If this is not the case, we would find three reflections of $F^{\prime}$ whose axes bound a hyperbolic triangle. This is impossible since $\bar{E}_{0}$ is not a hyperbolic triangle.

If the axis of $T^{\prime}$ does not intersect the boundary of $E_{0}^{\prime}$ there are again two possibilities: First there may be two reflections of $\varphi\left(r\left(E_{0}\right)\right)$ separated by $T^{\prime}$. This is shown to be impossible as above. Or then all the axes of the reflections of $\varphi\left(r\left(E_{0}\right)\right)$ are in the same component of $E^{1} \backslash \operatorname{Ax}\left(T^{\prime}\right)$. This means that if $R^{\prime}$ and $S^{\prime}$ are two reflections of $\varphi\left(r\left(E_{0}\right)\right)$, then $S^{\prime}$ does not separate $R^{\prime}$ and $T^{\prime}$. Using $D$ we can conclude that if $R, S \in$ $r\left(E_{0}\right)$, then $S$ does not separate $R$ and $T=\varphi^{-1}\left(T^{\prime}\right)$. Since for any reflection $R \in r\left(E_{0}\right)$, there is another $S \in r\left(E_{0}\right)$ such that the axes of $S$ and $R$ do not intersect, we would have that for any $S \in r\left(E_{0}\right), \operatorname{Ax}(T)$ and $E_{0}$ are in the same component of $E^{1} \backslash \operatorname{Ax}(S)$, and, consequently $\operatorname{Ax}(T) \cap E \subset E_{0}$ which is impossible.

Above we have assumed that $\bar{E}_{0}$ is not a hyperbolic triangle. If $\bar{E}_{0}$ is a hyperbolic triargle, then $S$ and $S^{\prime}$ are compact and, by $A, \operatorname{Fix}(F)$ and $\operatorname{Fix}\left(F^{\prime}\right)$ are dense in $S^{1}$. By proposition 3.5 we would have the homeomorphism $\varphi^{*}: S^{1} \rightarrow S^{1} \quad$ satisfying $\varphi^{*}(T(x))=\varphi(T)\left(\varphi^{*}(x)\right)$ for $x \in S^{1}$ and $T \in F$. If $R, S \in r\left(E_{0}\right), R \neq S$, the angle of $\bar{E}_{0}$ whose sides are sfgments of the axes of $R$ and $S$ is $2 \pi / k$ where $k$ is the order of the group generated by $S$ and $T$. Using the homeomorphism $\varphi^{*}$ we can now deduce that the angle of the triangle $\bar{E}_{0}^{\prime}$ whose sides are segments of axes of $\varphi(R)$ and $q(S)$ is $2 \pi / k$. Thus the triangles $\bar{E}_{0}$ and $\bar{E}_{0}^{\prime}$ would have equal angles and so they would be congruent in the sense of hyperbolic geometry (cf. e.g. Meschkowski [3]), i.e. there would be a conformal mapping $T$ of the unit disk such that $\bar{E}_{0}=T\left(\bar{E}_{0}^{\prime}\right)$ and hence $F_{0}=T F_{0}^{\prime} T^{-1}$. Since the group $F$ generated by $r\left(E_{0}\right)$ and $F_{0}$ does not contain reflections whose axes intersect $E_{0}$, the group generated by $\varphi\left(r\left(E_{0}\right)\right)$ and $F_{0}^{\prime}$, i.e. $F^{\prime}$, does not contain reflections whose axes intersect $E_{0}^{\prime}$.

It is an immediate consequence of $I$ that $E_{0}^{\prime}$ is a component of $p^{\prime-1}\left(S^{\prime} \backslash \partial S^{\prime}\right)$ where $p^{\prime}: E \rightarrow S^{\prime}$ is the canonical projection. Hence, we may identify $\bar{E}_{0}^{\prime} / F_{0}^{\prime}$ with $E^{\prime} \mid F^{\prime}=S^{\prime}$. The homeomorphism $f$ constructed in $H$ now defines a homeomorphism $g:\left(S, n_{F}\right) \rightarrow\left(S^{\prime}, n_{F^{\prime}}\right)$ of pointed surfaces. Let $z$ be a point of $E_{0}$ such that $n_{F}(p(z))=1$. Then by proposition 2.4 there is a unique homeomorphism $h: E \rightarrow E$ lifting $g$ such that $h(z)=f(z)$. The homeomorphism $h$ induces an isomorphism $\varphi^{\prime}: F \rightarrow F^{\prime}$. We must only prove that $\varphi^{\prime}$ equals $\varphi$. Clearly $h \mid E_{0}=f$ and from this it follows by $H$ that $\varphi\left|F_{0}=\varphi^{\prime}\right| F_{0}$ and $\varphi \mid r\left(E_{0}\right)=$ $\varphi^{\prime} \mid r\left(E_{0}\right)$. Since $r\left(E_{0}\right)$ and $F_{0}$ generate $F$, it follows that $\varphi$ equals $\varphi^{\prime}$. Thus the theorem is completely demonstrated. 
3.6.1 Corollary. Let $F$ and $F^{\prime}$ be two discrete groups of conformal mappings of the unit disk with compact fundamental domains and such that $F$ contains reflections. Then any isomorphism $\varphi: F \rightarrow F^{\prime}$ is induced by a homeomorphism.

Proof: Using A it is seen that $\operatorname{Fix}(F)$ is dense in $S^{1}$ and, consequently, $F$ contains a free subgroup with two generators. Then theorem 3.6 is applicable if we can prove that the relation of being crossed is preserved with every isomorphism. But this is seen easily using and idea of Nielsen in [5]. Since the proof depends only on the compactness of fundamental domains and not on the assumption that $F$ contains reflections, we give it in the form of a lemma.

3.6.2 Lemma. Let $F$ and $F^{\prime}$ be two discrete groups of conformal mappings of the unit disk with compact fundamental domains. Then any isomorphism $q: F \rightarrow F^{\prime}$ preserves the relation of being crossed.

Proof: It suffices to show that if $S$ and $T$ are two hyperbolic transformations of $F$ then $S^{\prime}=\varphi(S)$ and $T^{\prime}=\varphi(T)$ are not crossed if $S$ and $T$ are not crossed. To prove this we choose a fundamental domain $D$ for $F$. Then the finite set

$$
A=\{T \in F: T(D) \cap D \text { is a common side }\}
$$

is a set of generators for $F$. We set $A=\left\{T_{i}: i \in I\right\}$ where $I$ is some finite index set such that $I \cap \mathbf{Z}=\varnothing$, for reasons apparent later. Let $T_{i}^{\prime}=\varphi\left(T_{i}\right)$, $i \in I$, and

$$
M=\max _{i \in I} d\left(T_{i}^{\prime}(0), 0\right)
$$

where $d$ is the hyperbolic metric. Then there is $M^{\prime}>0$ such that if $d(T(0), 0)>M^{\prime}$ then $d(\varphi(T)(0), 0)>2 M$ for $T \in F$. Now it is easy to see that there are two mappings $n \rightarrow T_{n}$ and $n \rightarrow S_{n}$ from $\mathbf{Z}$ to $F$ such that if $\mathrm{X}$ is $S$ or $T$ we have

(i) $X_{n}^{-1} X_{n+1} \in A$ for all $n \in \mathbf{Z}$.

(ii) For every pair $m, n$ of integers $d\left(T_{m}(0), S_{n}(0)\right)>M^{\prime}$

(iii) There is $n_{X}>0$ such that $X_{n \div n_{X}}=X X_{n}$ for all $n \in \mathbf{Z}$.

We define $S_{n}^{\prime}=\varphi\left(S_{n}\right)$ and $T_{n}^{\prime}=\varphi\left(T_{n}\right)$ for $n \in \mathbf{Z}$. $S^{\prime}$ and $T^{\prime}$ are two hyperbolic transformations and by proposition 3.1 we have that $\operatorname{Ax}\left(T^{\prime}\right)$ $\neq \operatorname{Ax}\left(S^{\prime}\right)$. Then $\operatorname{Ax}\left(T^{\prime}\right) \cap \operatorname{Ax}\left(S^{\prime}\right)=\varnothing$ for otherwise there would be by (i) and (iii) two integers $n, m \in Z$ such that $d\left(T_{n}^{\prime}(0), S_{m}^{\prime}(0)\right)<2 M$. But this contradicts (ii).

Department of mathematics, University of Helsinki 


\section{References}

[1] Ahlfors, L. V. - Sario, L.: Riemann Surfaces - Princeton University Press, New Jersey, 1960

[2] Ford, L. R.: Automorphic Functions - Chelsea Publishing Co., New York, 1951

[3] Macbeath, A. M.: The classification of non-Euclidean plane crystallographic groups - Can. J. Math. 6, 1967

[4] Meschkowski, H.: Nichteuklidische Geometrie - Friedr. Vieweg \& Sohn, Braunschweig-Berlin-Stuttgart, 1954

[5] Nielsen, J.: Untersuchungen zur Topologie der geschlossenen zweiseitigen Flächen Acta Math. 50, 1927

[6] Zieschang, H.: Ueber Automorphismen ebener diskontinuierlicher Gruppen Math. Ann. 166, 1966

[7] Zieschang, H. - Vogt, E. - Coldewey, H.-D.: Flächen und ebene diskontinuierliche Gruppen - Lecture Notes in Mathematics 122, Springer-Verlag, Berlin-Heidelberg-New York, 1970

\section{Corrigendum}

The proof of the assertion $D$ in theorem 3.6 on p. 36 is incorrect. The last sentence in the paragraph following assertion $D$ should be replaced by the following sentence: This is seen using proposition 1.4 and the fact that either the axis of a reflection of $P^{\prime}$ of $F$ is the axis of a hyperbolic transformation of $F$ or every neighbourhood of an endpoint of the axis of $P^{\prime}$ contains axes of reflections of $F$. 